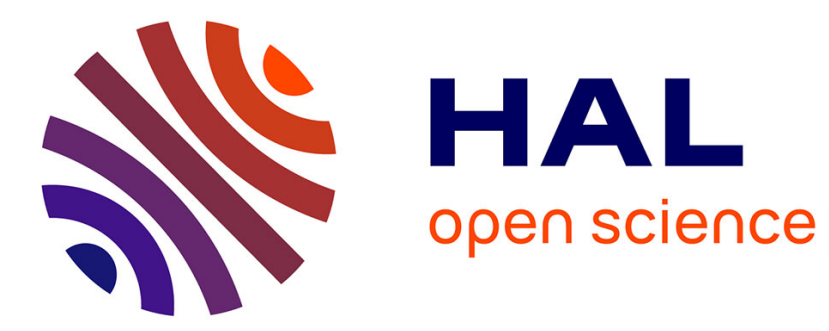

\title{
Improved energy-aware strategies for periodic real-time tasks under reliability constraints
}

Li Han, Louis-Claude Canon, Jing Liu, Yves Robert, Frédéric Vivien

\section{To cite this version:}

Li Han, Louis-Claude Canon, Jing Liu, Yves Robert, Frédéric Vivien. Improved energy-aware strategies for periodic real-time tasks under reliability constraints. [Research Report] RR-9259, Inria Research Centre Grenoble - Rhône-Alpes. 2019, pp.1-38. hal-02056520

\section{HAL Id: hal-02056520 \\ https://hal.inria.fr/hal-02056520}

Submitted on 4 Mar 2019

HAL is a multi-disciplinary open access archive for the deposit and dissemination of scientific research documents, whether they are published or not. The documents may come from teaching and research institutions in France or abroad, or from public or private research centers.
L'archive ouverte pluridisciplinaire HAL, est destinée au dépôt et à la diffusion de documents scientifiques de niveau recherche, publiés ou non, émanant des établissements d'enseignement et de recherche français ou étrangers, des laboratoires publics ou privés. 


\section{Improved energy-aware} strategies for periodic real-time tasks under reliability constraints

Li Han, Louis-Claude Canon, Jing Liu, Yves Robert, Frédéric Vivien

\section{RESEARCH}

REPORT

$\mathbf{N}^{\circ} 9259$

February 2019

Project-Team ROMA 



\title{
Inizía
}

\section{Improved energy-aware strategies for periodic real-time tasks under reliability constraints}

\author{
Li Han*†, Louis-Claude Canon ${ }^{\ddagger}$, Jing Liu ${ }^{\dagger}$, Yves Robert*\$, \\ Frédéric Vivien* \\ Project-Team ROMA
}

Research Report n 9259 - February 2019 - 38 pages

\begin{abstract}
This paper revisits the real-time scheduling problem recently introduced by Haque, Aydin and Zhu in a recent issue of this journal [9]. We provide new scheduling strategies that dramatically improve the results of [9].
\end{abstract}

Key-words: real-time systems, independent tasks, stochastic execution times, reliability, scheduling, energy-aware systems.

* LIP, École Normale Supérieure de Lyon, CNRS \& Inria, France

$\dagger$ East China Normal University, Shanghai, China

‡ FEMTO-ST, Université de Bourgogne Franche-Comté, France

$\S$ University of Tennessee Knoxville, USA

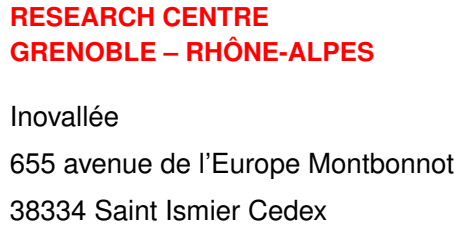




\section{Ordonnancement de tâches périodiques avec contraintes de fiabilité: minimisation de l'énergie}

Résumé : Ce rapport s'intéresse à un problème d'ordonnancement en temps-réel étudié par Haque, Aydin and Zhu et récemment paru dans IEEE TPDS [9]. Nous proposons de nouvelles stratégies de placement et d'ordonnancement qui améliorent significativement les résultats de [9].

Mots-clés : systèmes temps-réel, tâches indépendantes, temps d'exécution stochastiques, reliabilité, ordonnancement, économies d'énergie 


\section{Introduction}

This work revisits the results presented by Haque, Aydin and Zhu in a recent issue of this journal [9]. We call [9] the reference paper throughout the text. The reference paper deals with the following optimization problem: given a set of real-time tasks subject to (possibly different) periodic deadlines, how to execute them on a parallel platform and match all deadlines while minimizing the expected energy consumption? The problem is complicated by the need to enforce some reliability threshold, which is a standard constraint in real-time systems. In Section 2 below, we provide all details about this optimization problem. We do not provide any further motivation for this study: instead, we refer the reader to the reference paper.

The rest of this paper is organized as follows. Section 2 provides a detailed description of the optimization problem, and of the different methods used in the reference paper to solve it. In a nutshell, the reference paper uses a three-step approach: (i) for each task, compute a set of replicas and their frequencies; (ii) map and statically schedule all replicas onto the platform; (iii) dynamically update the schedule based on actual completion times (instead of worst-case ones) and observed successful executions. These three steps are described in Section 2.2 to 2.4. Then Section 3 outlines new propositions for each step, as well as a new complexity result that establishes the combinatorial nature of the scheduling step when the mapping is given. Section 3.4 summarizes new heuristics, while Section 3.6 deals with complexity results. Section 4 is devoted to a comprehensive experimental comparison of the results of the reference paper against those obtained with our improved approach. Section 5 presents related work, with the aim of complementing the related work already covered in the reference paper: we focus on recent works which quote the reference paper and briefly discuss their contributions. Finally, Section 6 gives concluding remarks and hints for future work.

\section{Optimization problem and previous approach}

In this section, we present the optimization problem in full details, and we describe the approach of the reference paper. Key notations are summarized in Table 1.

\subsection{Optimization problem}

The inputs to the optimization problem in the reference paper are a set of real-time tasks, a set of processors and a reliability target. More precisely:

Tasks - We have a set of $n$ periodic real-time tasks $\tau_{1}, \tau_{2}, \ldots, \tau_{n}$. Each task $\tau_{i}$ has worst-case execution time (WCET) $c_{i}$ under the maximum avail- 
able frequency $f_{\max }$. Note that real-time tasks actually complete execution earlier than their estimated WCET: execution times are assumed to be data-dependent and non-deterministic, randomly sampled from some probability distribution whose support is upper bounded by the WCET. Task $\tau_{i}$ generates a sequence of instances with period $p_{i}$, which is equal to its deadline. The whole input pattern repeats every hyperperiod of length $L=\operatorname{lcm}_{1 \leq i \leq n} p_{i}$. Each task $\tau_{i}$ has $\frac{L}{p_{i}}$ instances within the hyperperiod.

Processors - The platform consists of $M$ homogeneous processors, each of them having a set $F$ of different frequencies ranging from $f_{\min }$ to $f_{\max }$. Without loss of generality, we normalize the frequencies to enforce $f_{\max }=1$. At frequency $f_{i}$, a processor needs up to $\frac{c_{i}}{f_{i}}$ seconds to complete an instance of task $\tau_{i}$. The utilization $u_{i, f_{j}}$ of task $\tau_{i}$ at frequency $f_{j}$ is defined as $u_{i, f_{j}}=\frac{c_{i}}{f_{j} p_{i}}$. The utilization of a processor is the sum of the utilizations of all tasks that are assigned to it.

Fault model - One considers transient faults, modeled by an exponential distribution with average arrival rate $\lambda$. The fault rate $\lambda$ increases when frequency is scaled down using DVFS. Letting $\lambda_{0}$ denote the fault rate at maximum frequency $f_{\max }$. Then the fault rate at frequency $f_{i}$ is $\lambda\left(f_{i}\right)=\lambda_{0} \times \exp ^{\frac{d\left(1-f_{i}\right)}{1-f_{\min }}}$, where $d$ is called the sensitivity factor; $d$ is a measure of how quickly the transient fault rate increases when the system supply voltage and frequency are scaled. At the end of execution, there is an $a c$ ceptance test to check the occurrence of soft errors induced by the transient faults. It is assumed that acceptance tests are $100 \%$ accurate. The duration of the test is included within the task WCET. The reliability of a task instance is the probability of executing it successfully, in the absence of transient faults. The reliability of a single instance of task $\tau_{i}$ (with WCET $c_{i}$ ) running at frequency $f_{j}$ is $R_{i}\left(f_{j}\right)=\exp ^{-\lambda\left(f_{j}\right) \frac{c_{i}}{f_{j}}}$.

Reliability threshold - One assumes that the reliability threshold for each instance of task $\tau_{i}$ is $\mathcal{R}_{i}$, which may be given as part of the input. The reference paper also deals with the case where a reliability threshold is given for the whole task system over an hyperperiod of length $L=\operatorname{lcm}_{1 \leq i \leq n} p_{i}$ : then the reliability of each instance of $\tau_{i}, \mathcal{R}_{i}$, is computed using the Uniform Reliability Scaling technique [14]. We have $\omega=\frac{\phi_{i, \text { target }}}{\hat{\phi}_{i}}$ for all $i$, where $\omega$ is the uniform probability of failure scaling factor (given as part of the input), $\phi_{i, \text { target }}$ is the failure probability of task $\tau_{i}\left(\phi_{i, \text { target }}=1-\mathcal{R}_{i}{ }^{h_{i}}\right)$ and $\hat{\phi}_{i}$ is the failure probability of task $\tau_{i}$ when a single replica at maximum frequency is executed $\left(\hat{\phi}_{i}=1-R_{i}\left(f_{\max }\right)^{h_{i}}\right.$, where $h_{i}$ is the number of instances of task $\tau_{i}$ in the hyperperiod). This leads to $\mathcal{R}_{i}=\sqrt[h_{i}]{1-\omega\left(1-R_{i}\left(f_{\max }\right)^{h_{i}}\right)}$. Now, given the reliability threshold $\mathcal{R}_{i}$ for each (instance of) task $\tau_{i}$, the question 
Table 1: Key Notations

\begin{tabular}{ll}
\hline Notation & Explanation \\
\hline$c_{i}$ & WCET for task $\tau_{i}$ under max. available frequency \\
$p_{i}$ & period (deadline) for each task instance of task $\tau_{i}$ \\
$h_{i}$ & number of instances of task $\tau_{i}$ in the hyperperiod \\
$f_{j}$ & $f_{j} \in F=\left\{f_{\min }, \ldots, f_{\max }=1\right\}$ \\
$u_{i, f_{j}}$ & utilization of task $\tau_{i}$ at frequency $f_{j}$ \\
$f_{\text {opt }(i)}$ & most energy-efficient frequency for task $\tau_{i}$ \\
$R_{i}\left(f_{j}\right)$ & reliability of one instance of task $\tau_{i}$ under $f_{j}$ \\
$\mathcal{R}_{i}$ & target reliability threshold for one instance of task $\tau_{i}$ \\
$w$ & uniform probability of failure scaling factor \\
$k_{i, f_{j}}$ & min. replica number for task $\tau_{i}$ under $f_{j}$ to meet $\mathcal{R}_{i}$ \\
$E\left(\tau_{i}, f_{j}, k_{i, f_{j}}\right)$ & energy cost for task $\tau_{i}$ with $k_{i, f_{j}}$ replicas under $f_{j}$ \\
\hline
\end{tabular}

is to determine how many replicas to use, and at which frequency to execute them, so that the reliability threshold $\mathcal{R}_{i}$ is enforced while energy consumption is kept minimal. Note that all replicas of a given task instance will have same execution time if run at same frequency, because they operate on the same data.

Optimization objective - The objective is to determine a set of replicas for each task and their execution frequencies, and to build a static schedule of length $L=\operatorname{lcm}_{1 \leq i \leq n} p_{i}$ (the duration fo the hyperperiod), where the replicas of each instance of each task $\tau_{i}$ are mapped onto the processors, so that energy consumption is minimized, while matching the deadline $p_{i}$ and reliability threshold $\mathcal{R}_{i}$ for each instance of each task $\tau_{i}$. We detail below how energy consumption is estimated. To further complicate matters, the static schedule is dynamically modified on the fly to take actual execution times rather than WCET into account. Also, as soon as one replica of a given task instance completes its execution successfully, all its other replicas become redundant and are terminated instantaneously.

\subsection{Replica sets}

In the reference paper, the first step of the approach is to construct a table with all information needed. For each task $\tau_{i}$, we try all possible frequencies between $f_{\min }$ and $f_{\max }=1$, and compute number of replicas needed, corresponding energy cost and CPU time.

Given a frequency $f_{j}$, we start by computing the number $k_{i, f_{j}}$ of copies that are needed for (each instance of) $\tau_{i}$. The reliability using a single task instance (no replica) is $R_{i}\left(f_{j}\right)=\exp ^{-\lambda\left(f_{j}\right) \frac{c_{i}}{f_{j}}}$. If $R_{i}\left(f_{j}\right) \geq \mathcal{R}_{i}$, the reliability threshold is enforced, and we need no replica, hence $k_{i, f_{j}}=1$. Otherwise, using $r$ additional replicas, the reliability increases to $R_{i}^{r}\left(f_{j}\right)=$ $1-\left(1-R_{i}\left(f_{j}\right)\right)^{r+1}$ (the task fails only if all $r+1$ copies fail), and we take 
the minimum value $r$ such that $R_{i}^{r}\left(f_{j}\right) \geq \mathcal{R}_{i}$. This leads to $k_{i, f_{j}}=r+1=$ $\left\lceil\frac{\log \left(1-\mathcal{R}_{i}\right)}{\log \left(1-R_{i}\left(f_{j}\right)\right)}\right\rceil$ with $\mathcal{R}_{i}=\sqrt[h_{i}]{1-\omega\left(1-R_{i}\left(f_{\text {max }}\right)^{h_{i}}\right)}$ (see Section 2.1). The reference paper maps different copies onto different processors, so necessarily $k_{i, f_{j}} \leq M$. If no value of $k_{i, f_{j}}$ can be found, frequency $f_{j}$ cannot be used, and a higher frequency must be selected.

Once we have determined the number $k_{i, f_{j}}$ of copies of $\tau_{i}$ at frequency $f_{j}$ (with $1 \leq k_{i, f_{j}} \leq M$ ), we compute the corresponding energy cost. The reference paper adopts a conservative strategy and sums up the energy cost of each copy. This is pessimistic because as soon as a copy is successful, the remaining copies are interrupted (if already started) or simply cancelled (if not started). Now, for each copy, the energy cost is estimated as the power times the execution time. We use $\frac{c_{i}}{f_{j}}$ for the execution time (which is an upper bound). As for the power $P\left(f_{j}\right)$ at frequency $f_{j}$, we use

$$
P\left(f_{j}\right)=P_{\text {static }}+P_{\text {dyn }}\left(f_{j}\right)=P_{\text {static }}+\left(P_{\text {indep }}+C \times f_{j}^{3}\right)
$$

where $P_{\text {static }}$ (the static power), $P_{\text {indep }}$ (the frequency-independent part of dynamic power) and $C$ (the effective switching capacitance) are systemdependent constants. Altogether, we derive the energy cost $E\left(\tau_{i}, f_{j}, 1\right)$ for one copy of task $\tau_{i}$ at frequency $f_{j}$ :

$$
E\left(\tau_{i}, f_{j}, 1\right)=P\left(f_{j}\right) \times \frac{c_{i}}{f_{j}}
$$

The final energy cost with $k_{i, f_{j}}$ copies is estimated as $E\left(\tau_{i}, f_{j}, k_{i, f_{j}}\right)=$ $k_{i, f_{j}} E\left(\tau_{i}, f_{j}, 1\right)$, summing over all copies. The total CPU time is then estimated as $S\left(\tau_{i}, f_{j}, k_{i, f_{j}}\right)=k_{i, f_{j}} \frac{c_{i}}{f_{j}}$.

Furthermore, each processor always consumes static power when idle (this consumption can be eliminated only by a complete shutdown). Hence we account for static power whenever the mapping and scheduling phases described below leave processors idle.

\subsection{Mapping and static schedule}

The first step provides an initial configuration as input to the second step, which is the mapping and static scheduling onto processors. The initial configuration consists of an assigned frequency and number of replicas for each (instance of each) task $\tau_{i}$. Given a configuration, the mapping builds a schedule for an hyperperiod of length $L=\operatorname{lcm}\left(p_{i}\right)$ as follows:

- sort the tasks by decreasing total CPU time, and renumber them so that

$$
S\left(\tau_{1}, f_{j(1)}, k_{1, f_{\text {opt }(1)}}\right) \geq S\left(\tau_{2}, f_{j(2)}, k_{2, f_{\text {opt }(2)}}\right) \geq \ldots
$$

- for $i$ ranging from 1 to $n$, successively map all $k_{i, f_{\text {opt }(i)}}$ copies of $\tau_{i}$ onto $k_{i, f_{\text {opt }(i)}}$ different processors, using the First-Fit Decreasing (FFD) bin 
packing heuristic [10]. When mapping all the $\frac{L}{p_{i}}$ instances of a given task copy on a processor (bin), we use the standard Earliest Deadline First (EDF) scheduling heuristic [11]. EDF tells us that a given processor (bin) is a fit for that copy if and only if the utilization of that processor does not exceed 1. Recall that the utilization of a processor is the sum of the utilizations of all task instances assigned to it.

Hence, for a given task, the mapping finds the first processor whose utilization makes it a fit for the first task copy (and all its instances). Then it finds the first next processor whose utilization makes it a fit for the second task copy (and all its instances), and so on.

If the mapping succeeds, we have built a static schedule for the hyperperiod. But it may well be the case that it is impossible to find a processor onto which to map a given task copy in the procedure, because all processor utilizations are too high to accommodate that copy. Then the reference papers proposes to enter an iterative procedure as follows:

- Change the initial configuration into the one where every task copy executes at maximal speed $f_{\max }=1$. This requires fetch the values $k_{i, f_{\max }}$ from the table and reordering the tasks by decreasing total CPU time $S\left(\tau_{i}, f_{\max }, k_{i, f_{\max }}\right)$.

- Apply the mapping heuristic (FFD mapping and EDF schedule) to the new configuration.

Now, if the latter mapping fails again, there is no solution, resource utilization is too high. However, if the mapping succeeds, its energy cost will be very high. The reference paper proposes a refinement scheme where some tasks are relaxed, meaning that their frequency is decreased down to its predecessor (going from their current value $f_{j}$ down to $f_{j-1}$ ). Initially, all tasks have frequency $f_{\max }$, and some tasks are greedily selected for relaxation. If relaxing a task $\tau_{i}$ fails to lead to a successful mapping, or if we have reached its energy-optimal frequency $f_{\text {opt }(i)}$, then $\tau_{i}$ is marked as ineligible. The scheme stops when all tasks become ineligible. The reference paper uses three different greedy criteria to pick up the next task to be relaxed among eligible tasks, Largest Energy First (LEF), Largest Power First (LPF), and Largest Utilization First (LUF). We refer to the reference paper for details. We have implemented the first two variants, LEF and LPF, because they are shown to outperform LUF in the reference paper.

\subsection{Dynamic schedule}

The static schedule, also called canonical schedule, is based upon the WCET of each task. It is never executed exactly as such, because the actual execution time of a task instance will be shorter than its WCET. Still, it is used as the baseline to guide dynamic updates. From the canonical schedule, each processor has an assignment list made up with all task instances that it has 
to execute during the hyperperiod.

Let us follow the operation of a given processor $P$. For simplicity, assume that $P$ computes the full EDF schedule for all tasks in its assignment list, during the entire hyperperiod. The reference paper uses a data structure, called Canonical Execution Queue (CEQ), to avoid the high cost of computing the static schedule, while preserving the same outcome. Recall that the EDF schedule uses the WCET of each task, and preemption, so that a given task instance may well be split into several chunks. Hence $P$ has an ordered list of chunks together with their starting and finish times in its EDF schedule.

Let $t$ be the starting time of the next chunk ch, from task instance $\tau$, to be processed by $P$ in its EDF schedule (initially, $t=0$ ). In the canonical schedule, this chunk executes in the interval $\left[t_{s}, t_{f}\right]$, where $t_{s}$ is the starting time and $t_{f}$ the finish time on $P$. Let $k$ be the number of copies of $\tau$ in the canonical schedule, distributed over $k$ different processors. A major idea of the reference paper is to differentiate the action of $P$ depending upon whether its own copy of $\tau$ is the first copy to start execution among the $k$ processors. So if the chunk ch is indeed the first chunk of any copy of $\tau$ to start execution, then $P$ promotes its copy of $\tau$ to the status of primary replica, and all the chunks of $\tau$ as primary chunks. $P$ signals the other $k-1$ processors that their copies (and their copy chunks) are secondary replicas (or chunks). Otherwise, the chunk ch has already been marked either as primary (if it is not the first chunk of $\tau$, but $\tau$ has been marked primary on $P$ previously) or as secondary (if some other processor has signalled $P$ previously). The action of $P$ is the following:

- if ch is a primary chunk, then $P$ starts its execution immediately, using the frequency given by the canonical schedule. This execution will last for a duration of $t_{f}-t_{s}$ seconds in the worst case

- if ch is a secondary replica, then $P$ executes it at frequency $f_{\max }$, and using ALAP (As Late As Possible) scheduling, as further detailed below

The rationale for executing secondary chunks at highest frequency $f_{\max }$ is that it allows for a minimal execution time, hence a maximal delay for ALAP scheduling. When delaying secondary chunks, we hope that the primary copy will complete before secondary copies actually start, hence will be cancelled whenever the primary copy succeeds. At least, this ALAP strategy should minimize overlap between primary and secondary copies, hence minimize redundant work. We point out that the choice for primary/secondary replicas is done dynamically, by the first processor to start a task instance in the actual execution of the hyperperiod. It may well be the case that two different instances of the same task have not the same primary processor.

There remains to explain in full details how secondary chunks are scheduled on $P$. In fact, all secondary chunks are delayed according to the finish time of $\tau$ in the canonical schedule. Recall that the canonical schedule pro- 
vides an execution interval $\left[t_{s}, t_{f}\right]$ for every chunk of $\tau$. Assume there are $m$ chunks. For notational convenience, let $\left[t_{s}(i), t_{f}(i)\right], 1 \leq i \leq m$ denote the $m$ execution intervals in the canonical schedule. The sum of these $m$ interval lengths $t_{f}(i)-t_{s}(i), 1 \leq i \leq m$ is equal to $\frac{c}{f}$, where $c$ is the WCET of $\tau$ and $f$ is its frequency from the canonical schedule. Since we have decided to execute $\tau$ at frequency $f_{\max }=1$, we only need $c$ seconds, in the worst-case, to execute $\tau$. For instance if $f=0.5$ we only need (at most) half the time planned in the canonical schedule. The reference paper uses backfilling and reserves a total of $c$ seconds in the dynamic schedule, starting from the last interval and going backwards to the first interval, allocating slots until $c$ seconds are reserved. Then $P$ will execute $\tau$ greedily using these intervals from the beginning until completion of $\tau$. Finally, all chunks of $\tau$ will be scheduled across $n$ new intervals $\left[t_{s}^{\prime}(i), t_{f}^{\prime}(i)\right]$, where $1 \leq i \leq n$ and $n \leq m$, because very likely $\tau$ will finish before its WCET. Here are two examples with $c=20, f=0.5$ and $m=3$ :

- the intervals in the canonical schedule are $[5,35],[40,46]$ and $[50,54]$. We reserve 20 seconds out of the 40 available in these three intervals by keeping the third interval entirely (4 seconds), then keeping the second interval entirely ( 6 seconds) and then keeping the last 10 seconds of the first interval. Then $P$ will use these reserved slots ([25, 35], $[40,46]$ and $[50,54])$ to execute its copy of $\tau$ at frequency $f_{\max }$ starting from time 25 .

- the intervals in the canonical schedule are $[10,18],[40,46]$ and $[50,76]$. We reserve 20 seconds out of the 40 available in the three intervals by just keeping the last fraction $[56,76]$ of the third interval (20 seconds), and leaving the first and second intervals empty.

In both cases, $P$ will consume the slots from the beginning of the first reserved interval, until consuming all time units needed to finish $\tau$, say at time $t^{\prime}\left(t^{\prime} \leq t_{f}^{\prime}(n)\right)$. Once the copy of $\tau$ on the processor $P$ is successfully executed, all other copies (chunks) of $\tau$ are removed from the EDF list on other processors. Then $P$ will start processing the next chunk $c h^{\prime}$ in its list right at time $t^{\prime}$ if $c h^{\prime}$ is primary, otherwise, the chunks are delayed using the same mechanism.

To summarize, consecutive primary replicas are scheduled ASAP (As Soon As Possible), while secondary replicas are scheduled ALAP, which aims at reducing the overlap between copies over different processors, so as to minimize energy consumption.

\section{New strategies}

We identify several possible reasons why the approach in the reference paper may be sub-optimal:

- All the optimizations in the dynamic schedule aim at reducing overlap 
among replicas, in order to avoid redundant work. In their final schedule, the primary replica of task $\tau_{i}$ is executed at assigned frequency $f_{j}$, but the frequency of all $k_{i, f_{j}}-1$ secondary replicas is increased to $f_{\text {max }}$. However the energy consumption is estimated with all replicas at frequency $f_{j}$. We revisit the estimate for energy consumption and use a different formula, with one copy executing at $f_{j}$, and the rest at $f_{\text {max }}$ : this is expected to be closer to actual execution scenarios;

- The mapping uses the First-Fit Decreasing (FFD) bin-packing heuristic, which is likely to create imbalance across processors. Instead we use the Worst-Fit Decreasing (WFD) bin-packing heuristic [6], which selects the least-loaded processor that is a fit for the current task copy. WFD has been shown to reduce imbalance in a related framework [2], and we use it with a similar motive;

- The mapping maps all copies of a task before proceeding to the next task. Instead, we map the first copy of each task, and then the second copy of each task (whenever it exists), and so on. This layered approach is expected to: (1) evenly map primary replicas onto processors; (2) decrease the overlap among copies of the same task as long as EDF priority constraints do not call for a full reordering of the tasks during (or after) the mapping. For instance, assume for simplicity that all tasks have same period, so that the schedule is not constrained by EDF. Then, the mapping of the reference paper will place all replicas of the first task at the beginning of the assignment list of the processors, with possibly more primary replicas on the first processors, while it is better to delay all copies but one and insert first copies of other tasks instead.

- For the dynamic schedule, we keep the idea of running one primary replica of an instance of task $\tau_{i}$ at assigned frequency $f_{j}$ and to execute all other replicas at frequency $f_{\max }$, but we implement several novel aggressive strategies to reorder and delay chunks, as described in Section 3.3;

We outline these modifications step by step below, in Sections 3.1, 3.2 and 3.3, which are the respective counterpart, with same title, of Sections 2.2, 2.3 and 2.4. Section 3.4 summarizes our new heuristics. Note that we have both online and offline scheduling heuristics. For online scheduling strategies, which are able to determine dynamically the primary copy and secondary copies on the fly, all copies are mapped onto processors using frequency $f_{j}$ : this is because we must reserve enough room for each copy in the mapping. In other words, we assume that each copy is a primary copy in the mapping, and some of them become secondary only during execution. On the contrary, for offline scheduling strategies, we have decided primary copy and secondary copies during the mapping phase, which means we map one replica at $f_{j}$ and the rest at $f_{\max }$. In both scenarios, recall that we use different formulas to estimate the energy cost in both cases. Finally, Section 3.6 is devoted to 
complexity results.

\subsection{Replica sets}

For task $\tau_{i}$ at frequency $f_{j}$, the reference paper determines the number of copies $k_{i, f_{j}}$ needed to match the reliability threshold $\mathcal{R}_{i}$ and estimates the energy cost as $k_{i, f_{j}} E\left(\tau_{i}, f_{j}, 1\right)$ where $E\left(\tau_{i}, f_{j}, 1\right)$ is the energy cost for a single copy. Instead, we propose to estimate the energy cost as:

$$
E\left(\tau_{i}, f_{j}, k_{i, f_{j}}\right)=E\left(\tau_{i}, f_{j}, 1\right)+\left(k_{i, f_{j}}-1\right) E\left(\tau_{i}, f_{\max }, 1\right)
$$

because secondary replicas are actually executed at $f_{\max }$ in the reference paper. Equation (1) is an accordance with the pessimistic scenario where no replica is cancelled.

Note that since we do not use the same estimation formula for the energy cost as in the reference paper, we may find a different frequency $f_{\text {opt }(i)}$ to be used for the mapping step. Again, only one copy (the primary copy) will actually be executed at frequency $f_{\text {opt }(i)}$, while all remaining copies (the secondaries) will be executed at frequency $f_{\max }$. For each task $\tau_{i}$ and a given primary frequency $f_{j}$, we determine the minimum number of replicas $r_{i}$ such that the reliability threshold $\mathcal{R}_{i}$ for each instance is met. Recall that the reliability using a single task instance (no replica) is $R_{i}\left(f_{j}\right)=\exp ^{-\lambda\left(f_{j}\right) \frac{c_{i}}{f_{j}}}$. If $R_{i}\left(f_{j}\right) \geq \mathcal{R}_{i}$, the reliability threshold is enforced, and we need no replica, hence $k_{i, f_{j}}=1$. Otherwise, using $r$ additional replicas at $f_{\text {max }}$, the reliability increases to $1-\left(1-R_{i}\left(f_{j}\right)\right)\left(1-R_{i}\left(f_{\max }\right)\right)^{r}$, instead of $1-\left(1-R_{i}\left(f_{j}\right)\right)^{r+1}$, and we take the minimal value of $r$ such that this reliability exceeds $\mathcal{R}_{i}=$ $\sqrt[h_{i}]{1-\omega\left(1-R_{i}\left(f_{\max }\right)^{h_{i}}\right)}$. This leads to $r=\left\lceil\frac{\log \left(\frac{1-\mathcal{R}_{i}}{1-R_{i}\left(f_{j}\right)}\right)}{\log \left(1-R_{i}\left(f_{\max }\right)\right)}\right\rceil$. The new value of $r$ may be smaller than before, because each replica is more reliable. Of course, the new value of $r$ leads to a new value of $k_{i, f_{j}}=r+1$. We use this new value of $k_{i, f_{j}}$ to compute the energy cost.

We retain the frequency $f_{\text {opt }(i)}$ that minimizes Equation (1), and we use $k_{i, f_{\text {opt }(i)}}$ copies of task $\tau_{i}$ all running at frequency $f_{\text {opt }(i)}$ as input to the mapping phase for all online scheduling strategies. On the contrary, for offline scheduling strategies, we use one copy at frequency $f_{\text {opt }(i)}$ and $k_{i, f_{\text {opt }(i)}}-1$ copies at $f_{\max }$ as we explained before. Note that we tried through all possible frequencies between $f_{\min }$ and $f_{\max }=1$, but the final number of valid frequency levels may be smaller than the number of available frequency levels. There are several possibilities: 1) We should not consider frequency levels that below $c_{i} / p_{i}$, as the task $\tau_{i}$ would miss its deadline. 2) As we decrease the frequency level, the number of required replicas may increase or remain the same, the energy consumption is not strictly decreasing. 3) A lower frequency may introduce more overlap that can not be avoided than a higher frequency, so we enforce that $c_{i} / f_{j}+c_{i} / f_{\max } \leq p_{i}$. 


\subsection{Mapping and static schedule}

We map each replica to a processor while ensuring that no two replicas of the same task are assigned to the same processor. The mapping is done for a whole hyperperiod, with the following constraint for each task: when the first iteration (in case the period differs from the hyperperiod) of a replica is assigned to a given processor, all the other iterations of the same replica will be assigned to the same processor. It allows a simple feasibility check based upon the utilization of all the replicas assigned to the processor.

Recall that any success of a primary replica leads to the immediate cancellation of the secondary replicas, a crucial source of energy saving. The objective of the proposed mapping is thus to avoid overlapping between the execution of the primary and secondary replicas for each task: the primary must be terminated as soon as possible, while the secondaries must be delayed as much as possible. To this end, the mapping strategy ventilates primaries on all processors, in order to minimize conflict among several primary executions. Moreover, it allocates the secondaries while leaving idle time on all processors. This slack can then be used at execution time to delay the execution of the secondaries.

As shown in Algorithm 1, given a list of primary and secondary replicas for each task, and their execution times, we first execute the Worst-Fit Decreasing (WFD) allocation on the primaries ordered by their total execution time (Line 2). For all secondaries, we assign the same frequency as the primary if preparing for online scheduling afterwards. We assign them the maximum frequency $f_{\max }$ otherwise (Lines 3-6). Then we execute WFD on the secondaries layer-by-layer and ordered by their total execution time (Line 7), which means we consider the first secondary of all tasks, then the second and so on (as long as it exists). If we successfully map all replicas onto the processors, and the sum of the utilization of each replica on each processor is less than or equal to one (see Section 2.3), then we return this allocation. Otherwise, we could not find a feasible mapping with this replica setting (Lines 8-11). We observed that the competitor strategy FFD in the reference paper tends to compact all replicas onto the minimum number of processors, while WFD spreads replicas among all available processors, which may give a higher static energy cost. To reduce the influence of static power, we first run WFD with the number of processors used by FFD. If WFD is not able to find a feasible mapping, then we increase the number of processors by one up to the total available number.

We use this allocation mechanism to determine the number of replicas and their frequencies for each task (see Algorithm 2). As described in Section 3.1, we already know for each task, at each frequency level, how many replicas are needed to meet the reliability threshold. If we can find a feasible mapping with each task at its energy-optimal frequency $f_{\text {opt }(i)}$, then we return this optimal setting (Lines 2-7). Otherwise, we check the 


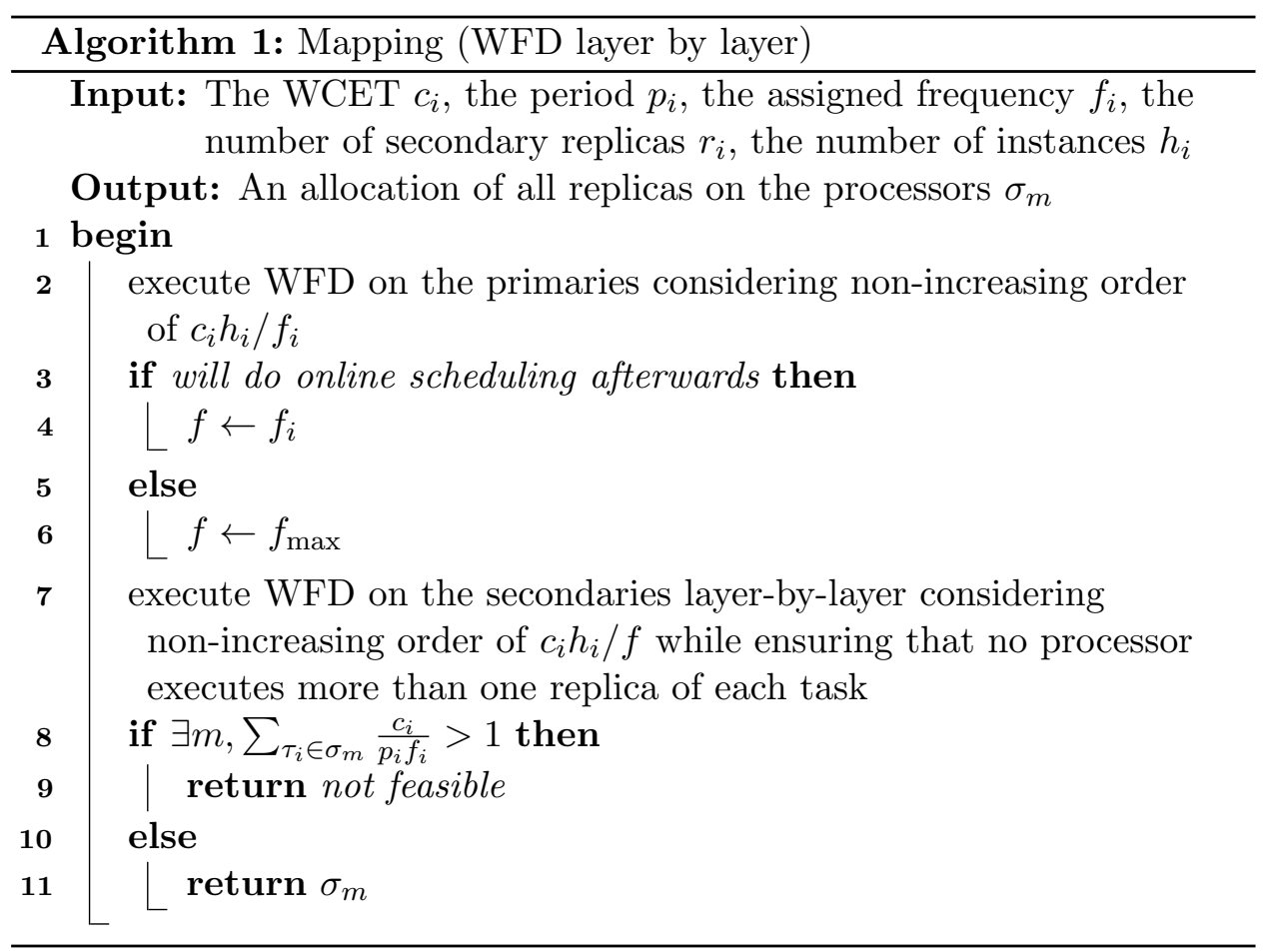

other end, all tasks run at $f_{\max }$ that takes the shortest time possible. If it is still impossible to map all replicas, then there does not exit a feasible mapping (Lines 8-13). If there exists a feasible mapping, we will enter the relaxing phase that decreases each primary frequency $f_{i}$ iteratively until it is no longer possible (Lines 14-19). Finally, we return the solution with a frequency level and number of secondary replicas for each task (Line 20).

\subsection{Dynamic schedule}

For the scheduling phase, it is important to start primary replicas as soon as possible, and to delay secondary replicas as much as possible, while still meeting all deadlines. As explained in Section 2.3, the reference paper uses the canonical schedule to compute the maximum delay for secondary replicas. Our improvements rely on the following techniques:

- Consider a scheduling interval defined by two consecutive deadlines in the global schedule. Inside the interval, task chunks to be executed are ordered by the EDF policy. We observe that we can freely reorder the chunks without missing any deadline, by definition of an interval. It means that in each interval, we should reorder to execute all primary replicas first, and then secondary replicas.

- It is possible to use only a fraction $\alpha$ of each scheduling interval, where 


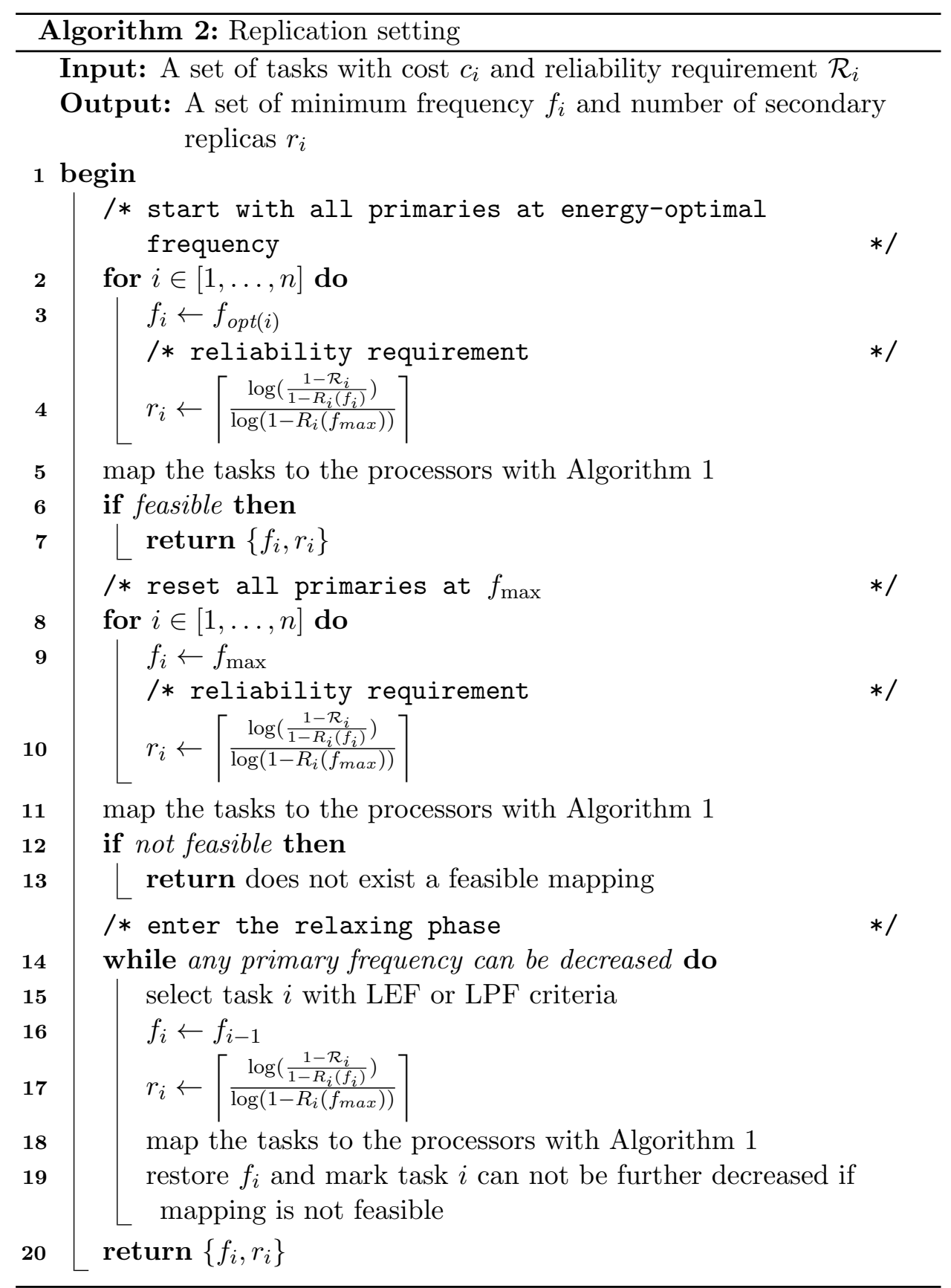


$\alpha$ is the utilization. Here is why: at the mapping phase, as long as the total utilization of replicas that are mapped onto the processor is less than or equal to one, then we are able to find a valid scheduling using EDF. Assume we have mapped three tasks $t_{i}, t_{j}, t_{k}$ onto processor $p$, and that the utilization is $\alpha=\frac{c_{i}}{p_{i} f_{i}}+\frac{c_{j}}{p_{j} f_{j}}+\frac{c_{k}}{p_{k} f_{k}}$. Either we keep the mapping and have a fraction $1-\alpha$ of the interval where $p$ is idle, or we slow down the execution time of all three tasks by a factor $\alpha$, then we will have a new utilization $\beta=\frac{c_{i}}{p_{i} f_{i} \alpha}+\frac{c_{j}}{p_{j} f_{j} \alpha}+\frac{c_{k}}{p_{k} f_{k} \alpha}=1$, which also gives us a feasible mapping without any idle time. This idea can be used in two ways:

1. Schedule while keeping a fraction $1-\alpha$ of idleness in each interval. Then, each primary replica is pushed to be beginning of the interval, while secondaries are pushed back to the end of the interval, with idleness in between.

2. Scale the WCET of all tasks by $\alpha$, which also gives a valid canonical schedule, but with longer worst case expected execution time for all tasks. This gives a better reference to further delay the start time of secondary replicas.

- Because we have delayed the start time of secondary replicas, there are some idle slots in the schedule. We take advantage of these idle slots by pre-fetching other primary replica chunks in the availability list: those primaries have been released but were scheduled later because they have lower EDF priority than the current secondary replicas.

\subsection{Heuristics}

Based on the above ideas, we propose several new scheduling heuristics which improve upon EDF_PAPER, the adaptive dynamic scheduling (and the most efficient) heuristic of the reference paper.

\subsubsection{EDF_Paper_PF}

This is an adaptive online scheduling that simply adds the pre-fetching mechanism to EDF_PAPER.

\subsubsection{EDF_Paper_PF_Utility}

This is an online scheduling heuristic where we refine EDF_PAPER_PF by using the utilization of each processor. We scale the worst case execution time of all replicas of a given processor by a factor $\alpha$, where $\alpha$ is the utilization of that processor. 


\subsubsection{EDF_Idle_Utility}

This is an offline scheduling that builds the EDF schedule for the whole hyperperiod. In each interval defined by two consecutive deadlines, we only use (on each processor) a fraction of the interval defined by the dynamic utilization, recomputed at each task completion and at the beginning of each interval. It consists in the following steps:

1. consider at each interval the EDF schedule, and keeping a fraction $1-\alpha$ of idle time

2. start the primary replicas and put aside the secondary replicas in a waiting list to be executed at the end of the interval. Note that for each secondary, as it is impossible to know the actual execution time before execution, we need to reserve the space for its WCET and to finish execution within the interval

3. fill in the idle period by inserting other primary replicas that are available

4. finish the execution of the interval execution with the secondary replicas in the waiting list, with their actual execution time

\subsubsection{EDF_Idle_CEQ}

This is an offline scheduling that only differs from EDF_IDLE_UtiLITY in that it uses the static utilization and step 1) is replaced by the following:

1. consider at each interval the EDF schedule with the constraint of keeping a fraction $1-\alpha$ of idle time. For secondaries, we refer to the canonical schedule to delay its start time without missing any deadline

\subsubsection{EDF_Idle_CEQ_Online}

This is the online version of EDF_IDLE_CEQ. It has two major advantages compared to the offline version: (1) we can dynamically decide the primary copy of each task instance, which gives us the flexibility to speed up replicas on the fly; (2) as long as we finish one replica successfully, we can safely cancel other replicas of the same task instance earlier than in static schedules, which enables us to have more flexibility to adjust the schedule afterwards. Moreover, we had to reserve the space for secondaries with their WCET which are usually larger than actual execution times, this dynamically generates some slots in the schedule, during which we also pre-fetch other available primary replica chunks. 


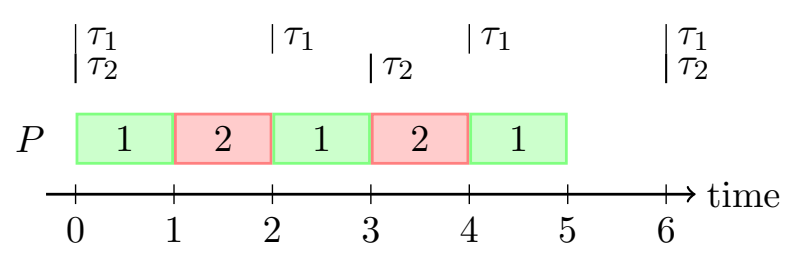

Figure 1: Canonical Execution Queue (CEQ) with two tasks $\tau_{1}$ and $\tau_{2}$ $\left(c_{1}=c_{2}=1, p_{1}=2\right.$ and $\left.p_{2}=3\right)$ on one processor $P$.

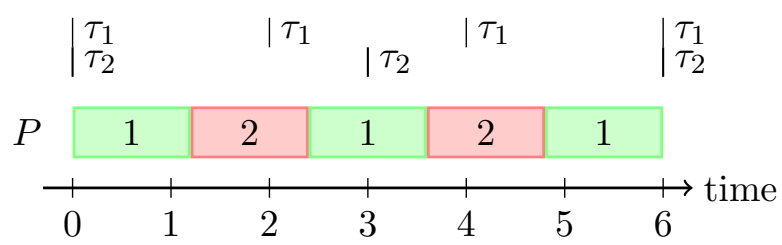

Figure 2: Canonical Execution Queue (CEQ) with scaled WCET with two tasks $\tau_{1}$ and $\tau_{2}\left(c_{1}=c_{2}=1, p_{1}=2\right.$ and $\left.p_{2}=3\right)$ on one processor $P$.

\subsection{Example}

We illustrate the difference between previous strategies with a simple example focusing one processor executing two tasks. Let $\tau_{1}$ and $\tau_{2}$ have WCET $c_{1}=c_{2}=1$ (the processor is assumed to run at maximum frequency) and periods $p_{1}=2$ and $p_{2}=3$. Figure 1 shows the CEQ in this case. Figure 2 depicts the CEQ computed with scaled WCET (for EDF_PAPER_PF_UTILITY).

For simplicity, we consider two scenarios for the primary selections. Either each instance of task $\tau_{1}$ is a primary (by an online decision or offline decision) and each instance of task $\tau_{2}$ is a secondary, or the contrary. We refer to the first scenario as $s_{1}$ and to the second as $s_{2}$. Also, the actual execution times are 0.5 with both tasks.

Figure 3 shows both scenarios with CEQ-based strategies (i.e. EDF_PAPER and EDF_PAPER_PF). Figure 4 exhibits improved scenarios (i.e. with more delay for the secondaries) when relying on the CEQ with scaled WCET (i.e. EDF_PAPER_PF_UTILITY). The idle-based mechanism (EDF_IDLE_UTiLITY) leads to the scenarios in Figure 5 and succeeds in executing $\tau_{2}$ first in the scenario $s_{2}$ because of the reordering mechanism. Finally, the idlebased mechanism with CEQ (EDF_IDLE_CEQ and EDF_IDLE_CEQ_ONLINE) leads to the scenarios in Figure 6.

\subsection{Complexity analysis}

This section is devoted to the proof of several complexity results for the scheduling phase. The global optimization problem is obviously NP-hard, 


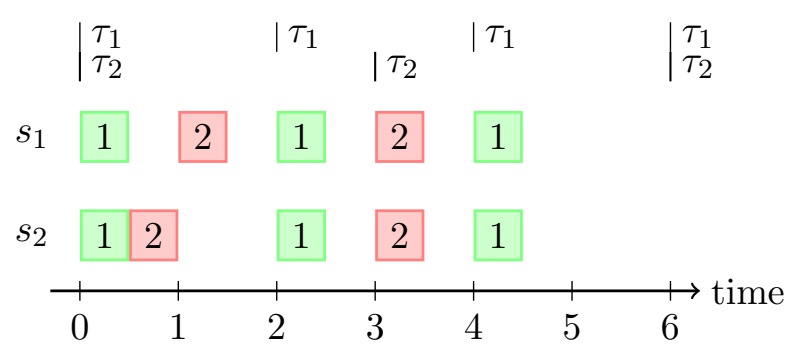

Figure 3: Executions when prioritizing primaries while delaying secondaries as in the Canonical Execution Queue (CEQ). There are two tasks $\tau_{1}$ and $\tau_{2}\left(c_{1}=c_{2}=1, p_{1}=2\right.$ and $\left.p_{2}=3\right)$ with actual execution times 0.5. In scenario $s_{1}$ (resp. $s_{2}$ ), $\tau_{1}$ is primary (resp. secondary) and $\tau_{2}$ is secondary (resp. primary).

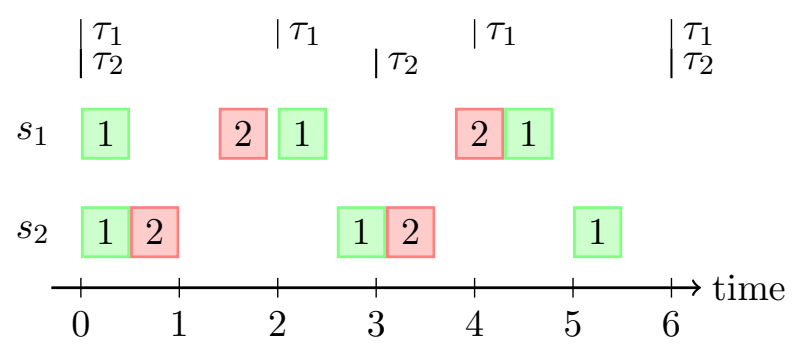

Figure 4: Executions when prioritizing primaries while delaying secondaries as in the Canonical Execution Queue (CEQ) with scaled WCET and with the same scenarios as in Figure 3.

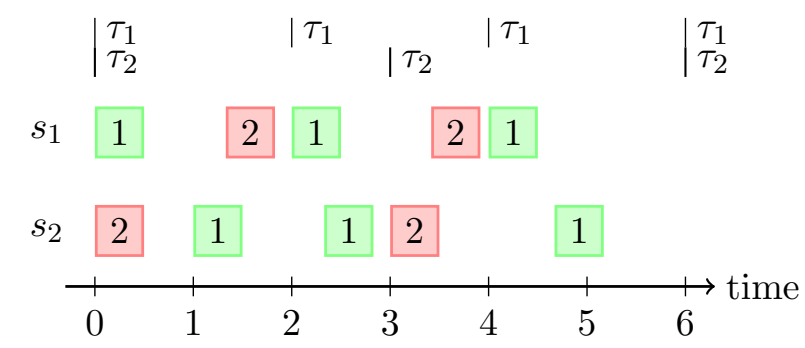

Figure 5: Executions when prioritizing primaries while delaying secondaries with idle time and with the same scenarios as in Figure 3. 


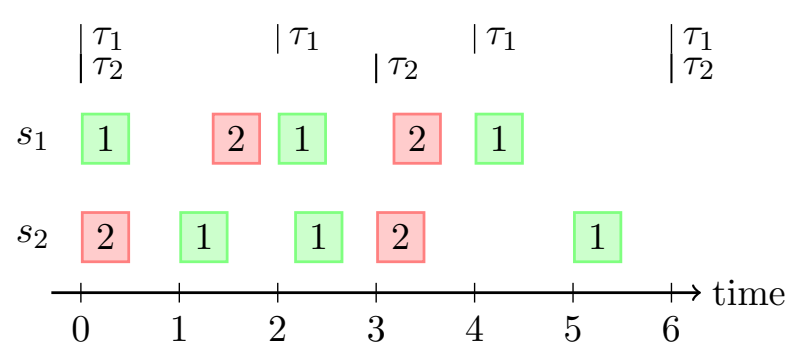

Figure 6: Executions when prioritizing primaries while delaying secondaries with idle time and with the Canonical Execution Queue (CEQ) and with the same scenarios as in Figure 3.

since it is a generalization of the makespan minimization problem with a fixed number of parallel processors [7]. However, the complexity of the sole scheduling phase is open: if the number or replicas has already been decided for each task, and if the frequency and assigned processor of each replica has also been decided, the scheduling phase aims at minimizing the expected energy consumption. We state a lower bound for this scheduling problem in Section 3.6.1, and we assess the complexity of achieving this lower bound: in Section 3.6.2, we show that the instance with identical WCETs is polynomial, while in Section 3.6.3, we show the instance with different WCETs is NP-complete in the strong sense.

\subsubsection{Lower bound}

Consider the following simple instance of the scheduling phase:

- All tasks have same period $p$, hence there is a single instance of each task in the hyperperiod of length $L=p$. hence EDF constraints do not apply, and each task is scheduled without preemption (as a single chunk)

- $F=1$ : there is a unique frequency $f_{\max }=1$

- For $1 \leq i \leq n$, task $\tau_{i}$ has $k_{i}$ replicas, including itself. The $j$-th replica, with $1 \leq j \leq k_{i}$, is mapped onto processor $P_{\text {alloc }(i, j)}$, where $1 \leq$ alloc $(i, j) \leq M$. For each task $\tau_{i}$, replicas are mapped onto different processors: $\operatorname{alloc}\left(i, j_{1}\right) \neq \operatorname{alloc}\left(i, j_{2}\right)$ for $1 \leq j_{1}<j_{2} \leq k_{i}$.

- The WCET of any replica of task $\tau_{i}$ is $c_{i}$, its reliability is $R_{i}=$ $R_{i}\left(f_{\max }\right)$, and its consumed energy is $E_{i}=E\left(\tau_{i}, f_{\max }, 1\right)$.

- $P_{\text {static }}=0$, meaning that no energy is spent when a processor is idle Thus, each of the $M$ processors has a list of assigned replicas to execute. It can choose any ordering because all tasks have same period, hence all deadlines will be enforced, regardless of the ordering. We further assume that the mapping is valid, which translates on each processor $P_{q}, 1 \leq q \leq M$, 
by the condition:

$$
\sum_{1 \leq i \leq n, 1 \leq j \leq k_{i}, \operatorname{alloc}(i, j)=q} W C E T_{i} \leq p
$$

Proposition 1. A lower bound on the total expected energy consumed is

$$
E_{L B}=\sum_{i=1}^{n}\left(E_{i} \sum_{j=1}^{k_{i}}\left(1-R_{i}\right)^{j-1}\right)
$$

The bound $E_{L B}$ is met if and only if the scheduling achieves no overlap between any two replicas of the same task.

Proof. For each task, we need to execute the replica which is scheduled in first position. If this replica fails, with probability $1-R_{i}$, we need to execute the replica which is scheduled in second position. If both replicas fail, we need to execute the replica which is scheduled in third position, and so on. This directly leads to the lower bound $E_{L B}$.

Now if any two replicas of the same task, say $\tau_{i}$, do overlap, then with some non-zero probability, both replicas will execute, and the consumed energy will be strictly higher than the contribution of $\tau_{i}$ to Equation (2). This concludes the proof.

\subsubsection{Identical costs}

Proposition 2. When all tasks have same $\operatorname{WCET}\left(c_{i}=c\right.$ for $1 \leq i \leq$ $n)$, one can build a schedule meeting the lower bound of Equation (2) in polynomial time.

Proof. We construct a bipartite graph with replicas on the left (with $K=$ $\sum_{i=1}^{n} k_{i}$ vertices) and processors on the right (with $M$ vertices). Each edge connects one replica to its assigned processor. We can assume that $K \geq M$ : if some processor is not used, simply discard it. Let $\delta$ be the maximum degree of a right vertex in the graph: this is the largest number of replicas assigned to a given processor. According to the weighted version of Konig's edge coloring theorem [12, Vol.A,Chapter.20], one can find a collection of $\delta$ perfect matchings that cover all edges in the graph, in time $O\left(K^{2}\right)$. Such a mapping directly leads to a schedule with minimal makespan $\delta c$. By construction, this schedule is guaranteed to be overlap-free.

\subsubsection{Arbitrary costs}

Proposition 3. When tasks have different WCET, determining whether the lower bound of Equation (2) can be met is a NP-complete problem. 
Proof. Let NoOverlap denote the problem with different WCETs. NoOvERLAP clearly belongs to the class NP: a certificate can be the description of the schedule with start and end times for each replica, and one can check in quadratic time that no two replicas of the same task overlap. We establish completeness through a reduction from 3-Partition [7]. Let $\mathcal{I}$ be an instance of 3-Partition. $\mathcal{I}$ comprises $3 m$ integers, $a_{1}, \ldots, a_{3 m}$ such that $\sum_{i=1}^{3 m} a_{i}=$ $m B$. The question is: can we partition the $a_{i}$ 's into $m$ subsets $S_{1}, \ldots, S_{m}$ such that each subset has total size $B: \sum_{j \in S_{i}} a_{j}=B$ ? The size of $\mathcal{I}$ is $O(m+B)$. From the instance $\mathcal{I}$ of 3 -Partition, we build an instance $\mathcal{J}$ of NOOVERLAP: this instance contains three types of tasks: some replicated tasks (the $R_{i}$ 's), some filling tasks which constrain the replicated tasks (the $F_{i, j}$ 's), and the tasks corresponding to the integers in instance $\mathcal{I}$ (the $A_{i}$ 's). Specifically:

- There are $1+m(m-1)$ processors denoted $P_{0}$ and $P_{i, j}$ with $1 \leq i \leq m$ and $1 \leq j \leq m-1$.

- All tasks have the same period $p=(2 m-1) B$.

- There are $m-1$ replicated tasks of size $B, R_{1}, \ldots, R_{m-1}$. For any $i$, $1 \leq i \leq m-1$, there is one replica of $R_{i}$ on processor $P_{0}$, and 1 on each of the processors $P_{k, i}, 1 \leq k \leq m$. Therefore, each $R_{i}$ 's is replicated $m+1$ times.

- Two tasks $F_{i, j, 1}$ and $F_{i, j, 2}$ are mapped on each processor $P_{i, j}, 1 \leq i \leq$ $m-1,1 \leq j \leq m . F_{i, j, 1}$ is of size $2(i-1) B$ and $F_{i, j, 2}$ is of size $2(m-i) B$. Therefore, the total load of processor $P_{i, j}$ is $B+2(i-1 * B+2(m-i) B=$ $(2 m-1) B$ and there is no slack on that processor. (Note that, to ease the writing, we have kept in our description a null size task on processors $P_{i, 1}$ and $P_{i, m}$.)

- In addition to one replica of each of the tasks $R_{1}, R_{m-1}, 3 m$ tasks $A_{1}, \ldots, A_{3 m}$ are mapped to processor $P_{0}$, where task $A_{i}$ has size $a_{i}$. Therefore, the total load of processor $P_{0}$ is $(m-1) B+\sum_{i=1}^{3 m} a_{i}=$ $(2 m-1) B$ and there is no slack on that processor either.

Instance $\mathcal{J}$ contains $1+m(m-1)$ processors and $(m-1)(m+1)+2 m(m-$ 1) $+3 m=3 m^{2}+3 m-1$ replica. Therefore, the size of $\mathcal{J}$ is polynomial in the size of $\mathcal{I}$.

We now prove that if $\mathcal{I}$ has a solution, then $\mathcal{J}$ has a solution. Let $S_{1}, \ldots, S_{m}$ be the solution of $\mathcal{I}$. Then we schedule the tasks of $\mathcal{J}$ as follows and as illustrated by Figure 7:

- For any $i, 1 \leq i \leq m-1$, and any $j, 1 \leq j \leq m$, on processor $P_{i, j}$, task $F_{i, j, 1}$ is executed during the interval [0,2(i-1)B], a replica of $R_{j}$ during the interval $[2(i-1) B,(2 i-1) B]$, and $F_{i, j, 2}$ during the interval $[(2 i-1) B,(2 m-1) B]$. 


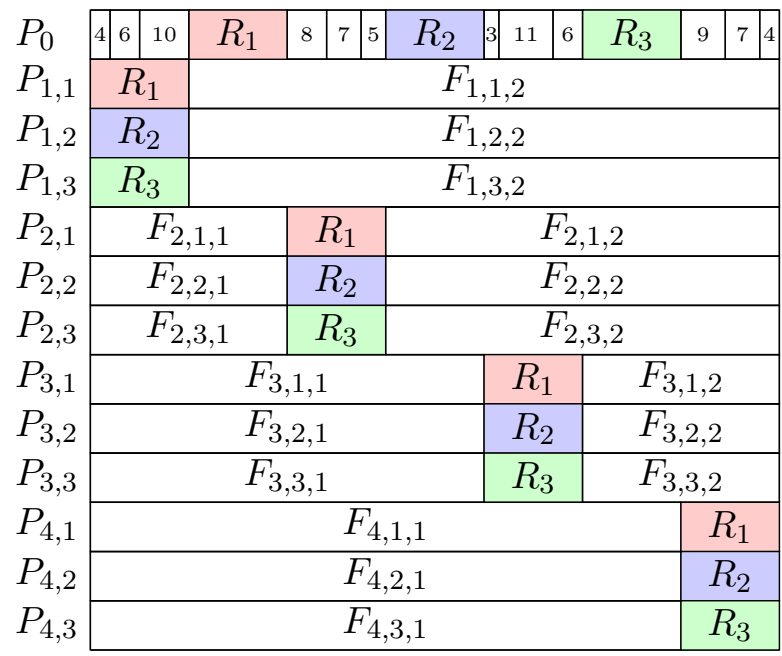

Figure 7: NP-completeness proof: scheduling for a solution of $\mathcal{I}$ of 3-Partition, with $m=4, B=20$, and $\left(a_{1}, \ldots, a_{12}\right)=$ $(4,3,8,9,7,6,11,6,7,10,4,5)$. On processor $P_{0}$ the digits are the sizes of the $A_{i}$ 's.

- For any $i, 1 \leq i \leq m-1$, a replica of $R_{i}$ is executed on $P_{0}$ during the interval $[(2 i-1) B, 2 i B]$.

- For any $j, 1 \leq j \leq m$, the tasks corresponding to the $j$-th partition of $\mathcal{I}$, i.e., the tasks $A_{k}$ such that $k \in S_{j}$, are executed on $P_{0}$ during the time interval $[2(j-1) B,(2 j-1) B]$.

One can easily check that this schedule is valid and that two replicas of a task $R_{i}$ are never executed simultaneously. Therefore there exists a schedule without overlap for $\mathcal{J}$ if there exists a solution for $\mathcal{I}$.

Let us now assume that there exists a valid schedule for $\mathcal{J}$, i.e., a schedule without any overlap. Let us consider any $i, 1 \leq i \leq m-1$. Processor $P_{i, 1}$ (respectively $P_{i, m}$ ) contains a replica $R_{i}$ and a task of size $(2 m-2) B$ (we do not care about the null-size task). Therefore, the replica $R_{i}$ is executed on $P_{i, 1}$ (resp. $\left.P_{i, m}\right)$ either during the interval $[0, B]$ or during $[(2 m-2) N,(2 m-$ 1) $B]$, Then, for any $j, 1<j \leq \frac{m}{2}$, processor $P_{i, j}$ (respectively $P_{i, m-j+1}$ ) contains a replica $R_{i}$, a task of size $(2 i-1) B$ and one of task $2(m-i) B$. From what precedes, there is already a replica of $R_{i}$ executed during the time interval $[0, B]$ and one during $[(2 m-2) B,(2 m-1) B]$. Therefore, the replica $R_{i}$ is executed on $P_{i, j}$ (resp. $P_{i, m-j+1}$ ) either during the interval $[(2 i-1) B, 2 i B]$ or during $[(2 m-i) B,(2 m-i+1) B]$. Overall, on processor $P_{0}$, the replica $R_{i}$ must be executed during one of the intervals $[(2 j-1) B,(2 j) B]$, for $1 \leq j \leq m-1$, because at all the other instants there is already one $R_{i}$ replica being executed on one other processor and because the schedule is without any overlap. However, there are $m-1$ such intervals and $m-1$ 
such replicas. Therefore, the tasks $A_{i}$ 's must be executed during the intervals $[(2 j-2) B,(2 j-1) B]$, for $1 \leq j \leq m$. This is a set of $m$ intervals each of size $B$. Because the schedule is valid all the $A_{i}$ 's are executed during these intervals. Let $S_{j}, 1 \leq j \leq m$, be the set of the indices of the $A_{l}$ 's executed during $[(2 j-2) B,(2 j-1) B]$. Then the subsets $S_{j}$ define a solution to $\mathcal{I}$.

\section{Performance evaluation}

In this section, we present the simulation results to evaluate the performance of our whole strategy compared to EDF_PAPER, the adaptive dynamic scheduling heuristic of the reference paper [9]. In Section 4.1, we describe the parameters and settings used during our experimental campaign. We present our results in Section 4.2.

\subsection{Experimental methodology}

We designed a discrete event simulator, which is publicly available at [8]. For each data point, we considered 2000 data sets with 20 tasks. Task periods are randomly generated with WCET between $10 \mathrm{~ms}$ and $100 \mathrm{~ms}$. The utilization of each task is generated randomly using the UUnifast scheme [3], with the total utilization $U_{t o t}$ as input. The number of normalized frequency levels is $F=10$ starting from to 0.1 to 1.0 . We assumed the coverage factor to be equal to 1 , because only with a perfect acceptance test, we are able to delete other replicas without missing the reliability target. Following [9], in the rest of the section, we assume that the transient fault arrival rate at $f_{\max }$ is $\lambda_{0}=10^{-6}$ and the system sensitivity factor $d=4$. The static power and the frequency-independent are set to $5 \%$ and $15 \%$ respectively of the maximum frequency-dependent power consumption with $C=1$. We use the ratio $B C / W C$ of the best-case (BC) over worst-case (WC) execution time, to model workload variability. The actual execution time of each task is determined according to a normal distribution, with the mean and the standard deviation set to $(B C+W C) / 2$ and $(W C-B C) / 6$ respectively. This guarantees the actual execution time is constrained in the range $[B C, W C]$ with probability $99.7 \%$. To compare the strategies under all parameter settings, we covered the whole range of all variables from [9]. For the rest of the section, we keep the number of cores at 8, vary the value of $U_{t o t}$ from 0.5 to 3.5 to study the impact of system load, vary the $B C / W C$ from 0.2 to 1.0 to show the influence of workload variability, and vary the probability of failure scaling factor $w$ from $10^{-5}$ to $10^{2}$ to evaluate the impact of target reliability. 


\subsection{Results}

We use the same baseline scheme as in the reference paper ${ }^{1}$, namely EDF, the classic EDF scheduling with FFD mapping and the replica sets from [9] (where it is called the static scheme). Figures 8 through 17 present the energy consumption of our strategies and of EDF_PAPER, divided by the energy consumption of the baseline. Therefore, the lower the better and data points below the $y=1$ line denote cases in which these strategies outperform the static scheme (i.e., achieve a lower energy consumption). Figures 8 through 12 consider heuristic LPF for choosing the candidate task for relaxation, while figures 13 through 17 apply heuristic LEF. We can see that different heuristics for relaxing phase do not influence the trends.

Each subfigure shows results for different combination of mapping and replica settings. Each line of subfigure is for a different replica set (REPLICASEt_Paper and ReplicaSet_OUR) while each column is for a different mapping (FFD and WFD). For example, the bottom right plot presents energy savings of several scheduling approaches (different line colors) with WFD mapping and our replica setting. We report on these figures the number of seeds (out of 2000 in total) that could find a feasible solution for each setting. These numbers are reported in black above the horizontal axis in each figure. We noticed that WFD tends to find less or equal number of feasible solutions compared to FFD. This is because FFD tries to pack more tasks onto processors while WFD tends to spread tasks onto less loaded processors. Moreover, we plot the lower bound for online scheduling and offline scheduling (different line styles) in black lines, by which we could know the maximum energy saving that can be achieved without any overlapping and failure. We can notice that when we apply REPLICASET_Our, the lower bound of the offline scheduling is lower that the online scheduling, the reason is that during the mapping phase, we assign all secondaries the same frequency as the primary if preparing for online scheduling, which makes it less possible to find a feasible mapping compared to the case for offline scheduling that assign secondaries the maximum frequency. So the later could have chance to further slow down the chosen frequency (see Section 3.2). This explains why sometimes the offline scheduling EDF_IDLE_CEQ outperforms the online version EDF_IDLE_CEQ_ONLINE.

A clear observation is that our scheduling heuristics outperform EDF_PAPER under almost all combination of settings, especially EDF_IDLE_CEQ_ONLINE always achieves the best performance when we apply REPLICASET_PAPER. When we apply RePlicaSet_Our, EDF_IdLE_CEQ is the best scheduling to go with low system utilization (see Fig. 8a), because its lower bound is lower than the online schedulings, and the possibility of finding a non-overlapping mapping to achieve its lower bound is high. But this possi-

\footnotetext{
${ }^{1}$ The authors of [9] have not provided their source code to us; we have done our best to ensure a fair assessment and comparison of results.
} 


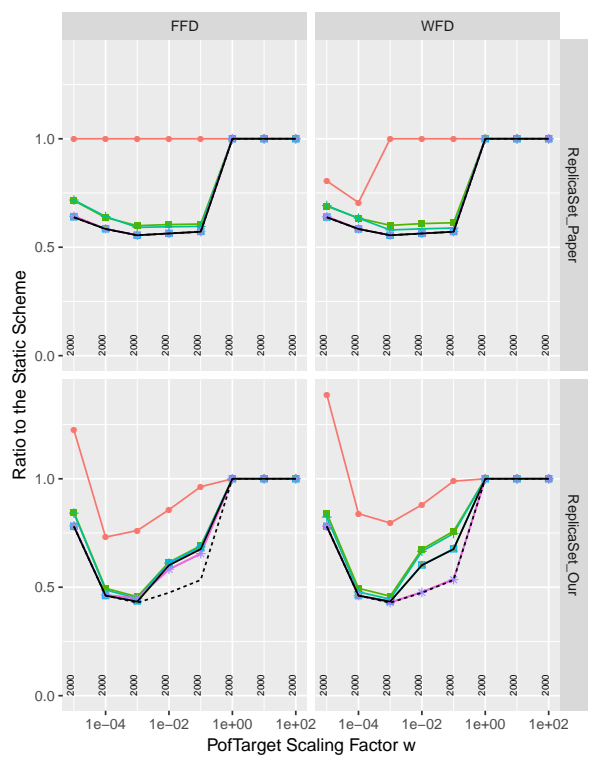

(a) $U_{t o t}=0.5$

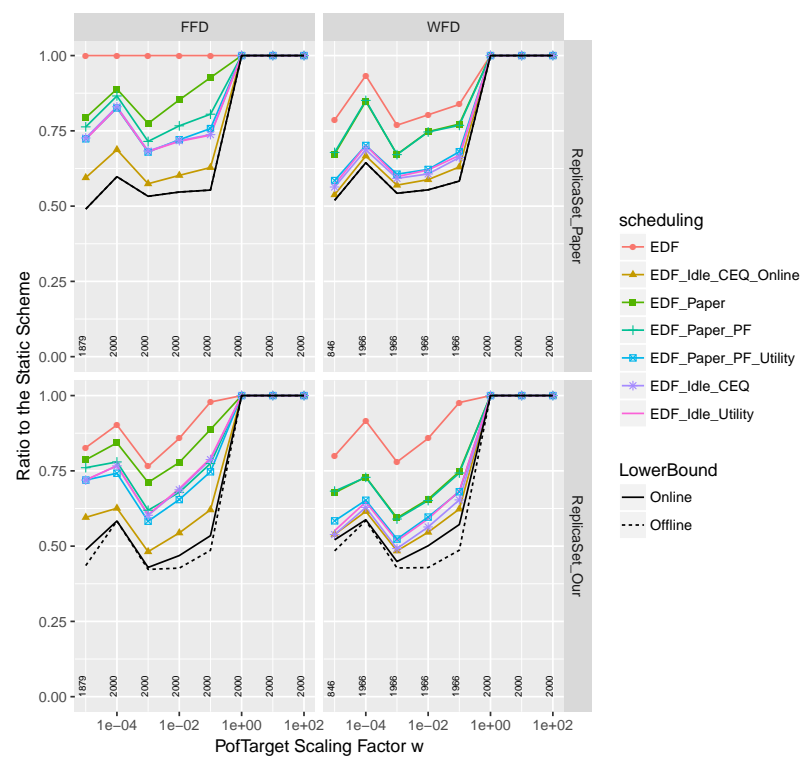

(c) $U_{t o t}=2.5$

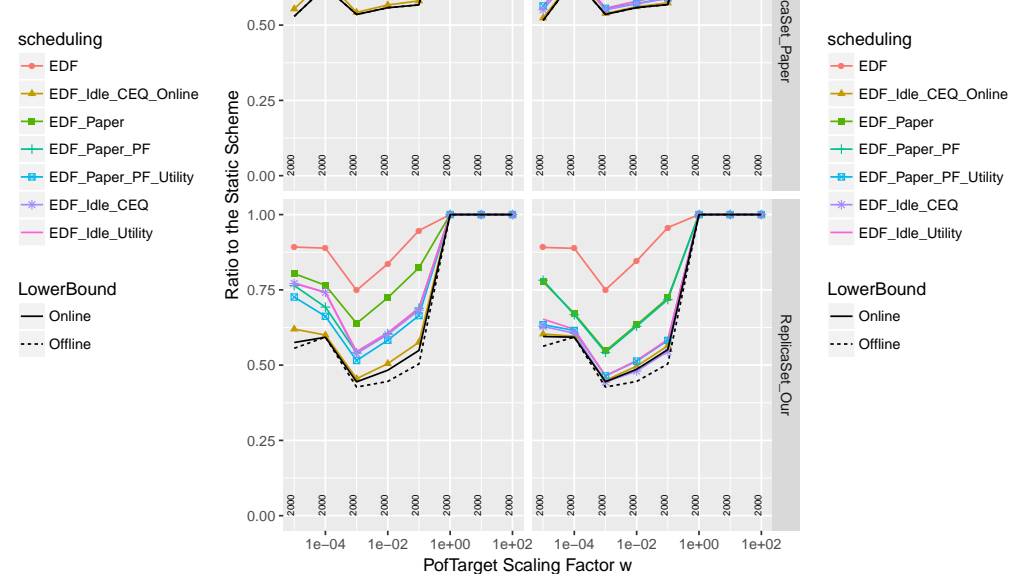

(b) $U_{t o t}=1.5$

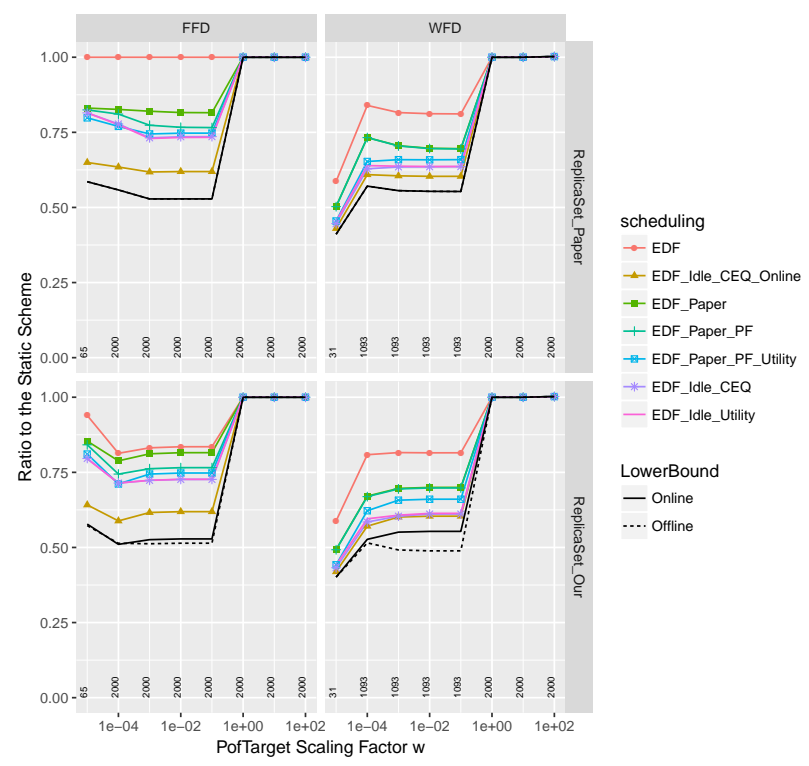

(d) $U_{t o t}=3.5$

Figure 8: Impact of $U_{\text {tot }}$ and $w$ with $B C / W C=1$ and LPF 


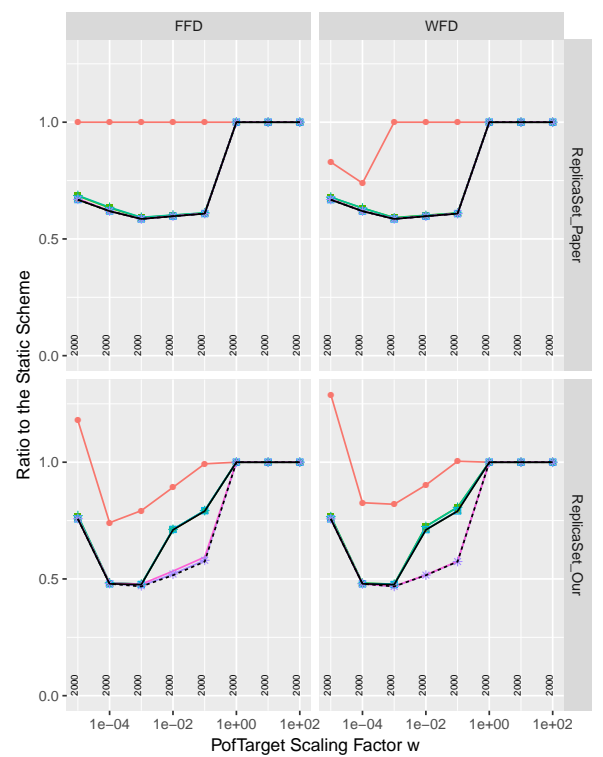

(a) $B C / W C=0.2$

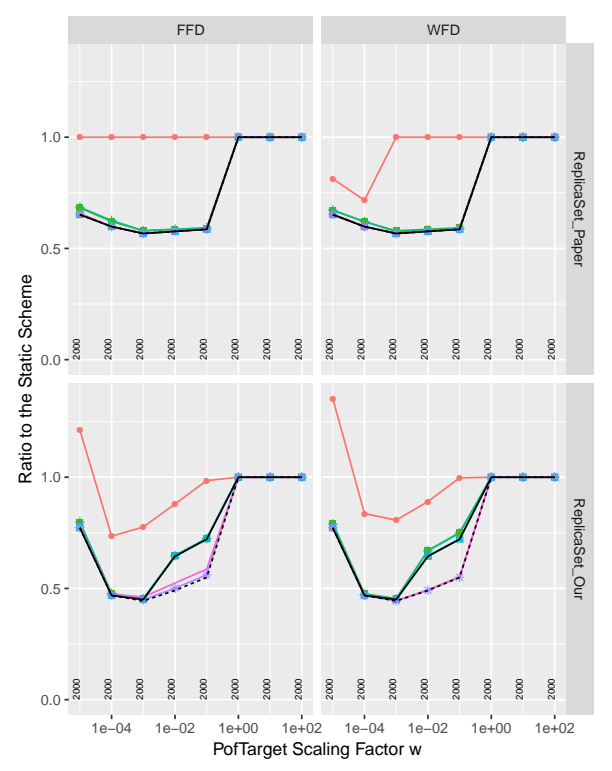

(c) $B C / W C=0.6$

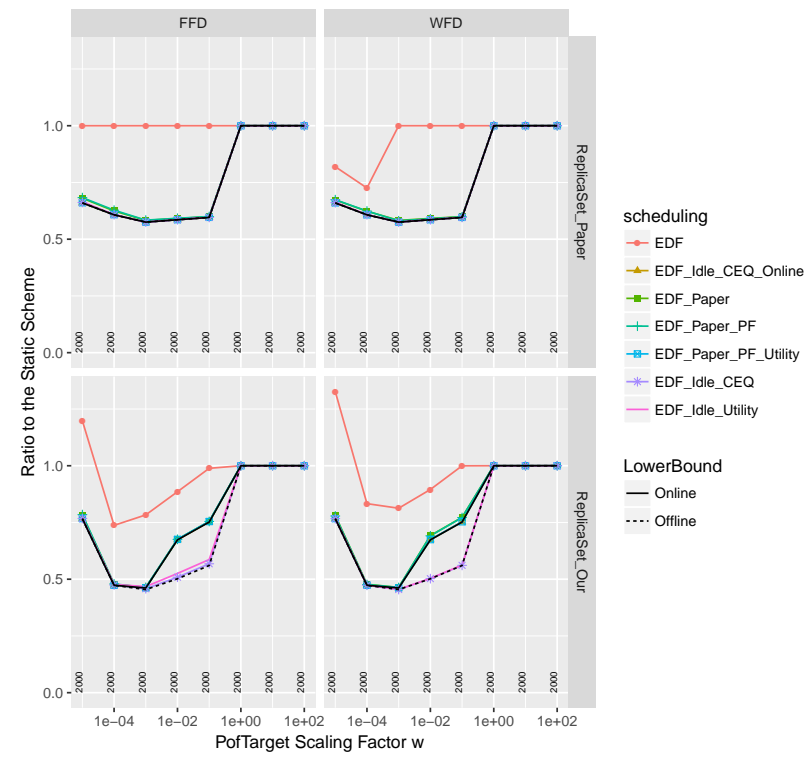

(b) $B C / W C=0.4$

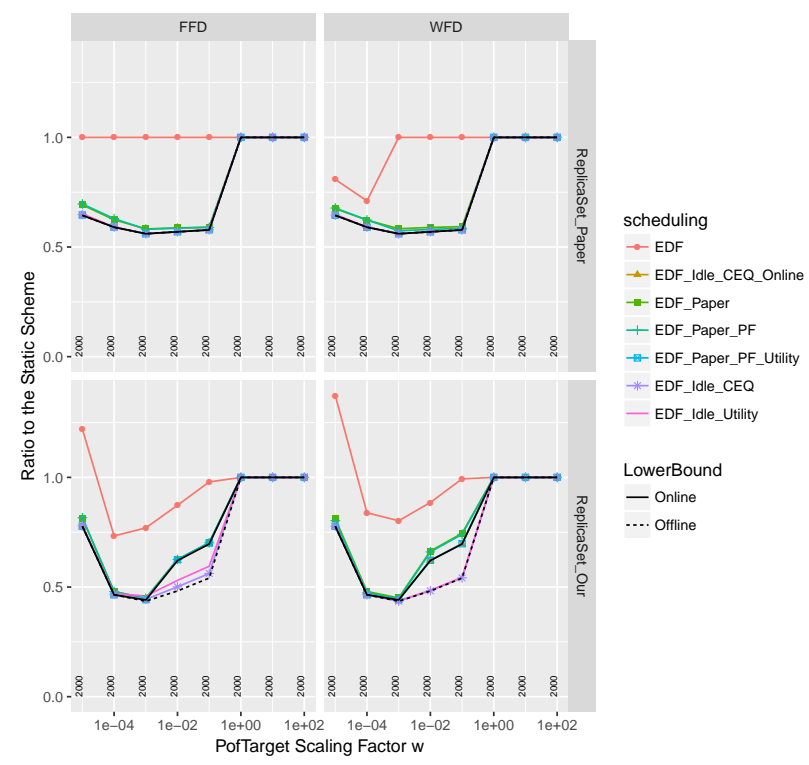

(d) $B C / W C=0.8$

Figure 9: Impact of $B C / W C$ and $w$ with $U_{\text {tot }}=0.5$ and LPF 


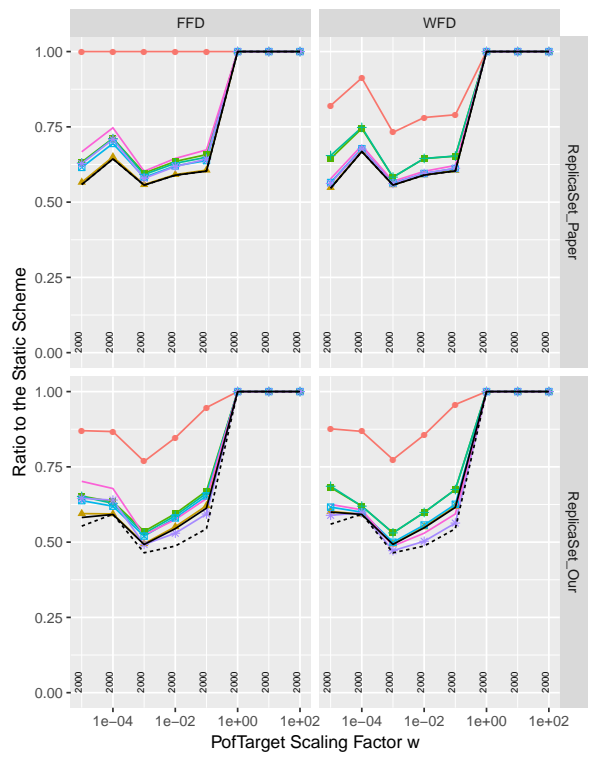

(a) $B C / W C=0.2$

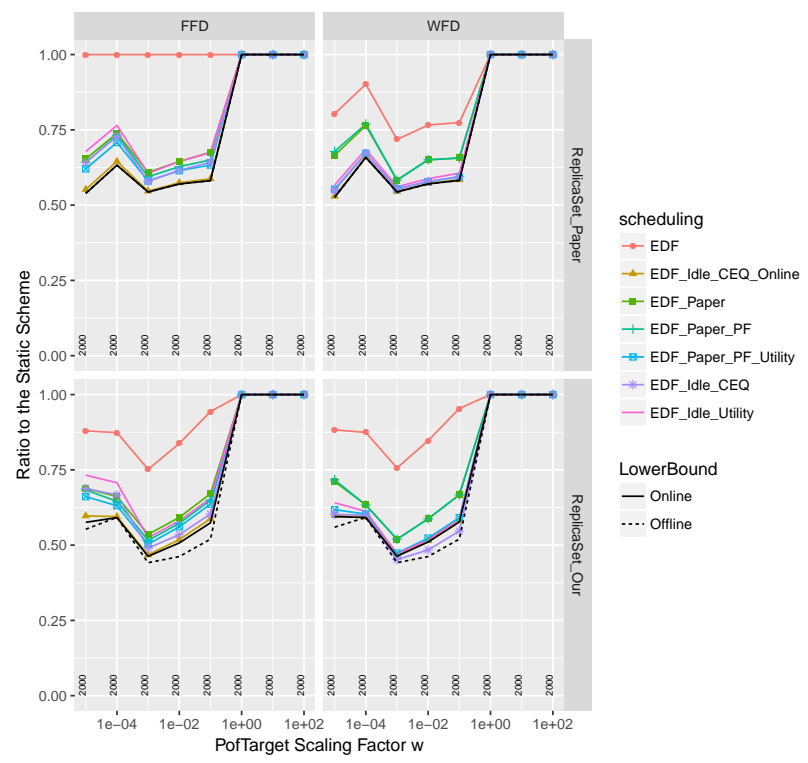

(c) $B C / W C=0.6$

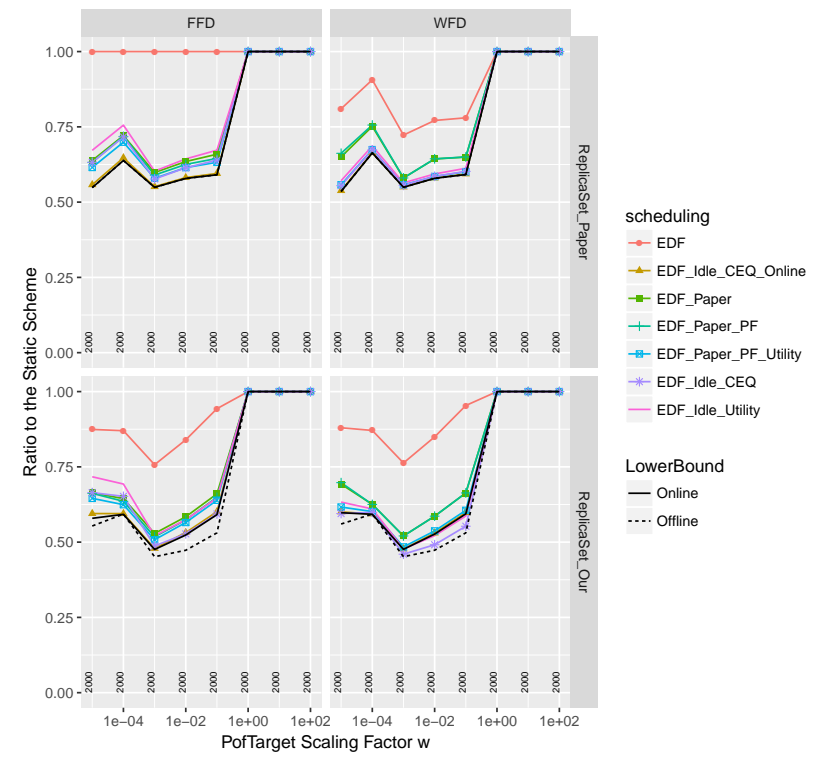

(b) $B C / W C=0.4$

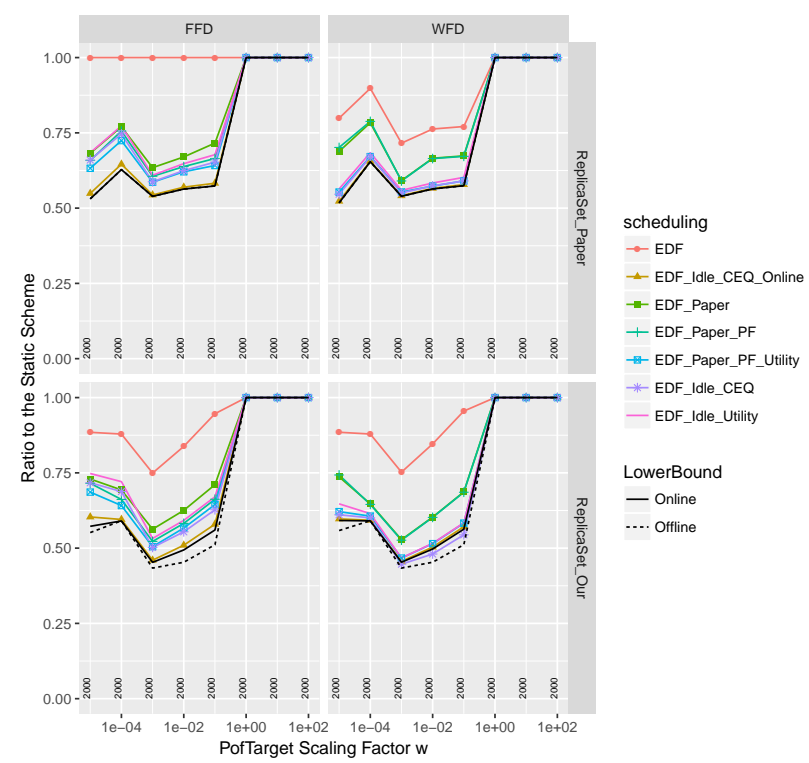

(d) $B C / W C=0.8$

Figure 10: Impact of $B C / W C$ and $w$ with $U_{t o t}=1.5$ and LPF 


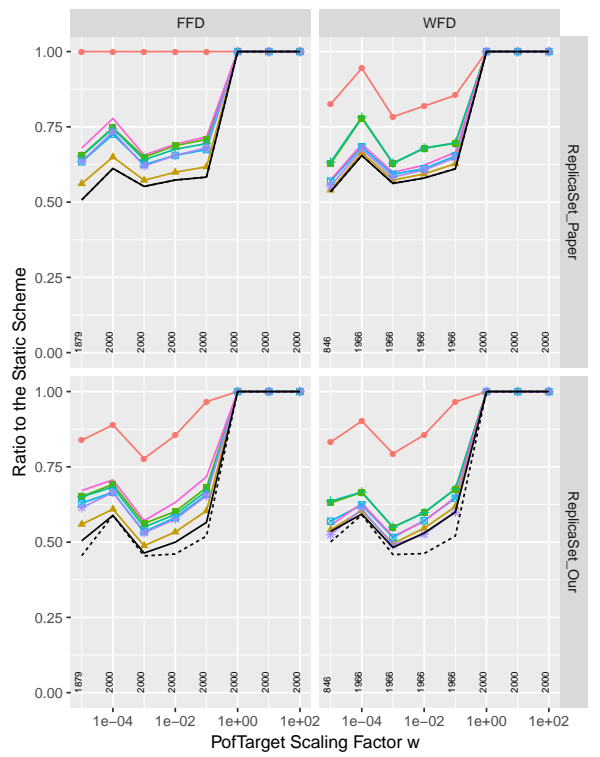

(a) $B C / W C=0.2$

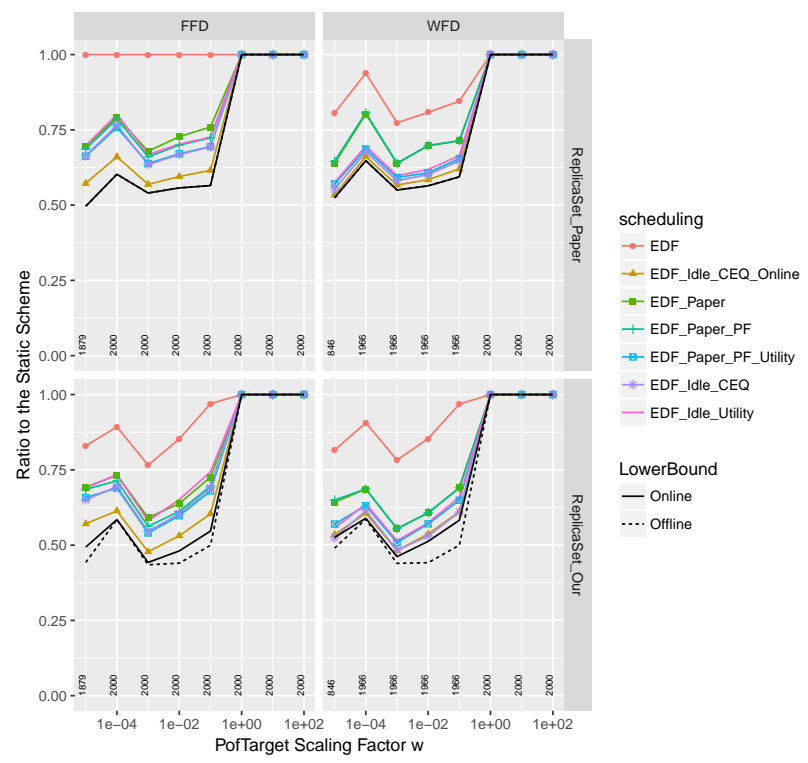

(c) $B C / W C=0.6$

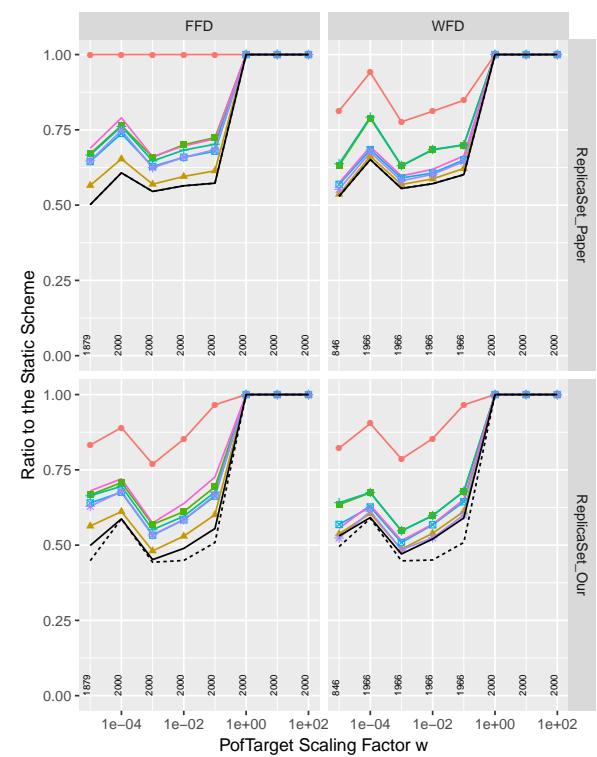

(b) $B C / W C=0.4$

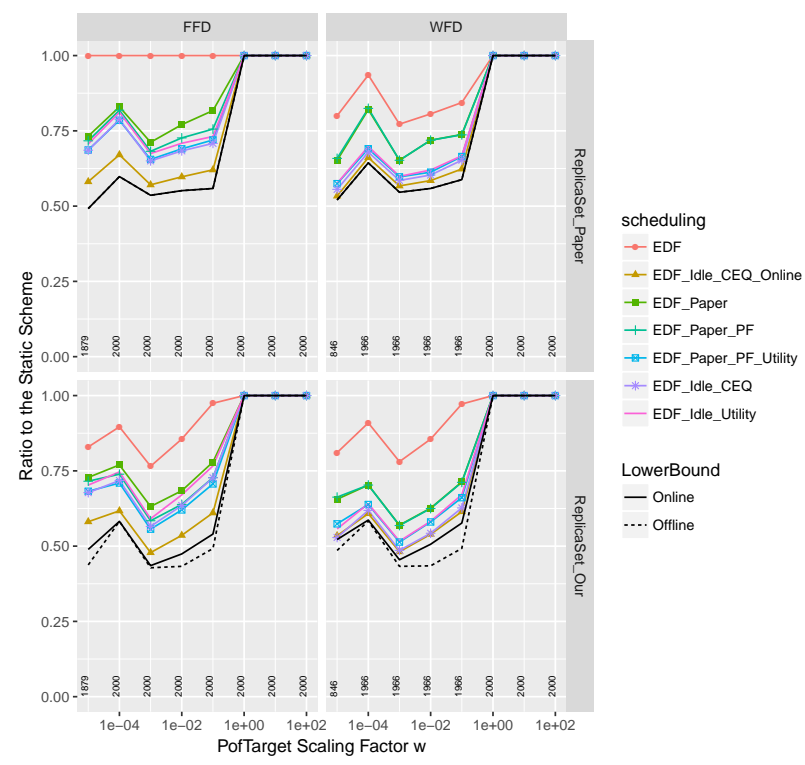

(d) $B C / W C=0.8$ scheduling

$\therefore$ - EDF

^-EDF_Idle_CEQ_Online

- EDF_Paper

+ EDF_Paper_PF

\#- EDF_Paper_PF_Utility

*-EDF_Idle_CEQ

- EDF_Idle_Utility

LowerBound

- Online
-... Offline

Figure 11: Impact of $B C / W C$ and $w$ with $U_{t o t}=2.5$ and LPF 


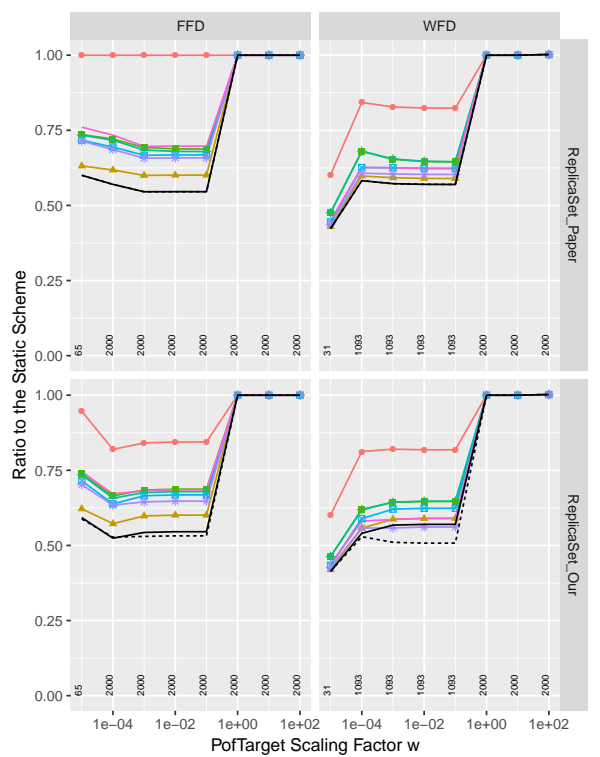

(a) $B C / W C=0.2$

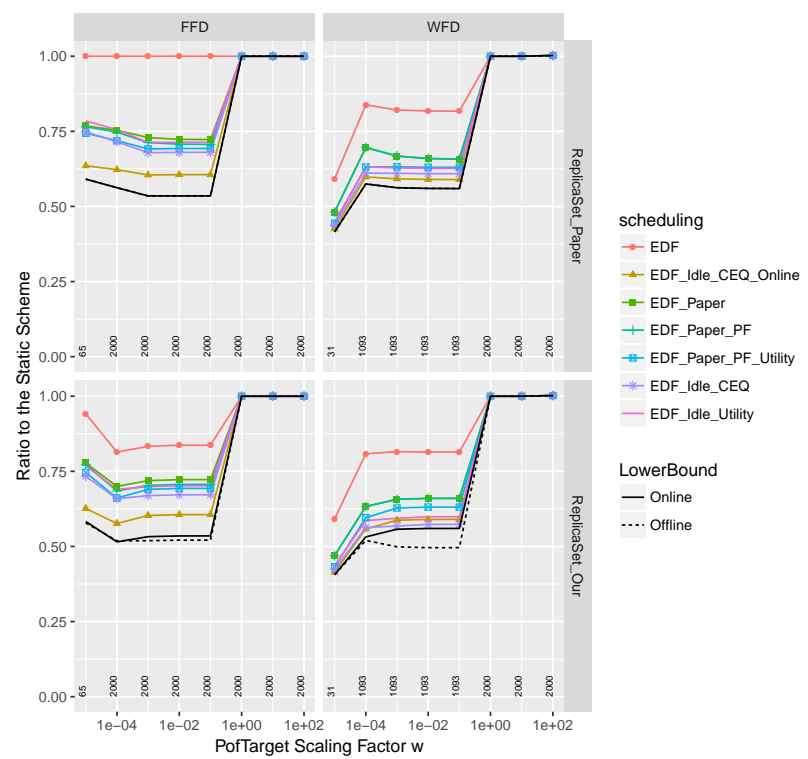

(c) $B C / W C=0.6$

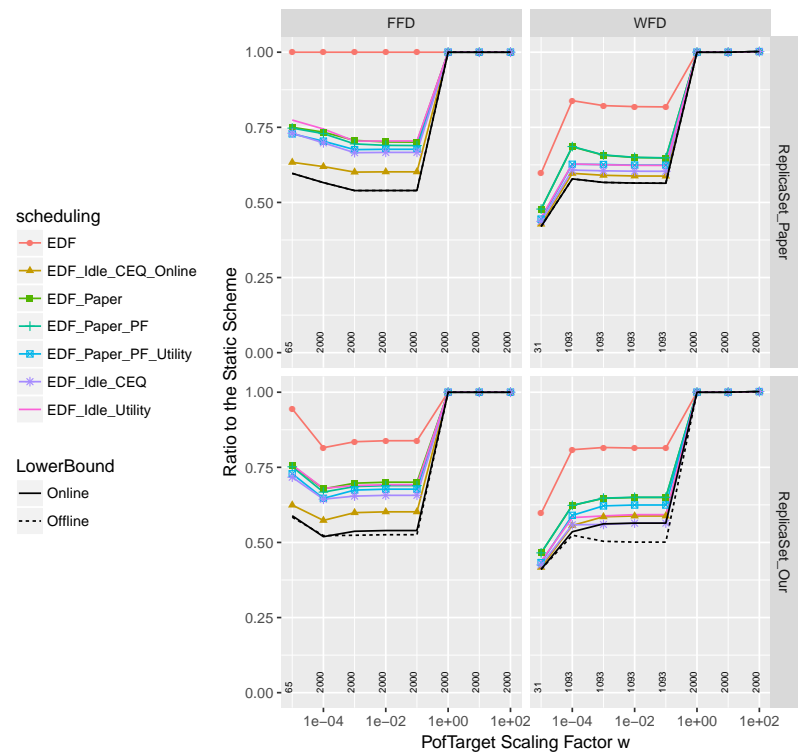

(b) $B C / W C=0.4$

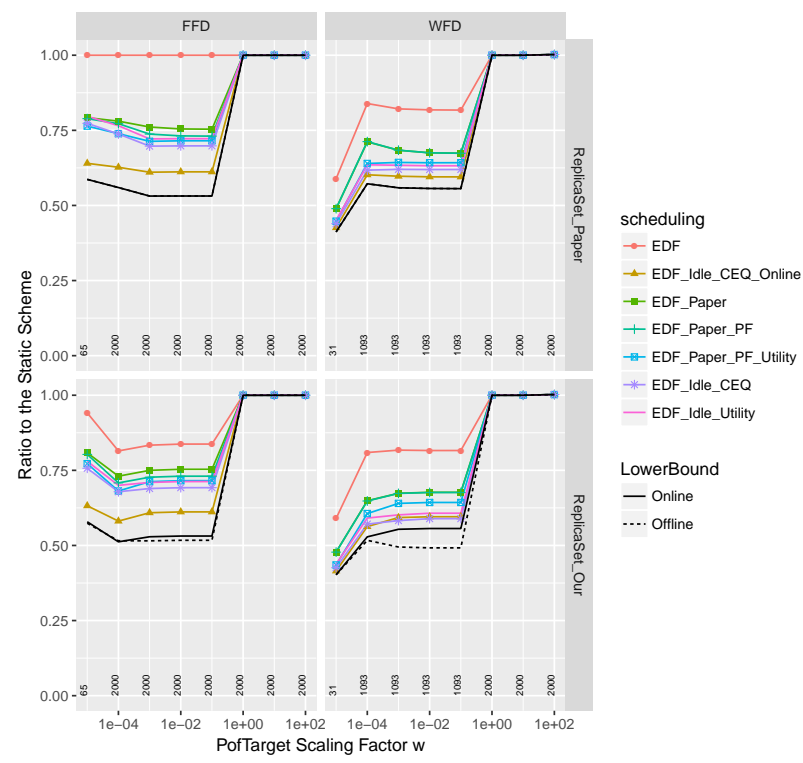

(d) $B C / W C=0.8$ scheduling

$\therefore$ - EDF

4-EDF_Idle_CEQ_Online

- EDF_Paper

+ EDF_Paper_PF

\#-EDF_Paper_PF_Utility

*-EDF_ddle_CEQ

- EDF_Idle_Utility

LowerBound

— Online

-... Offline

Figure 12: Impact of $B C / W C$ and $w$ with $U_{\text {tot }}=3.5$ and LPF 


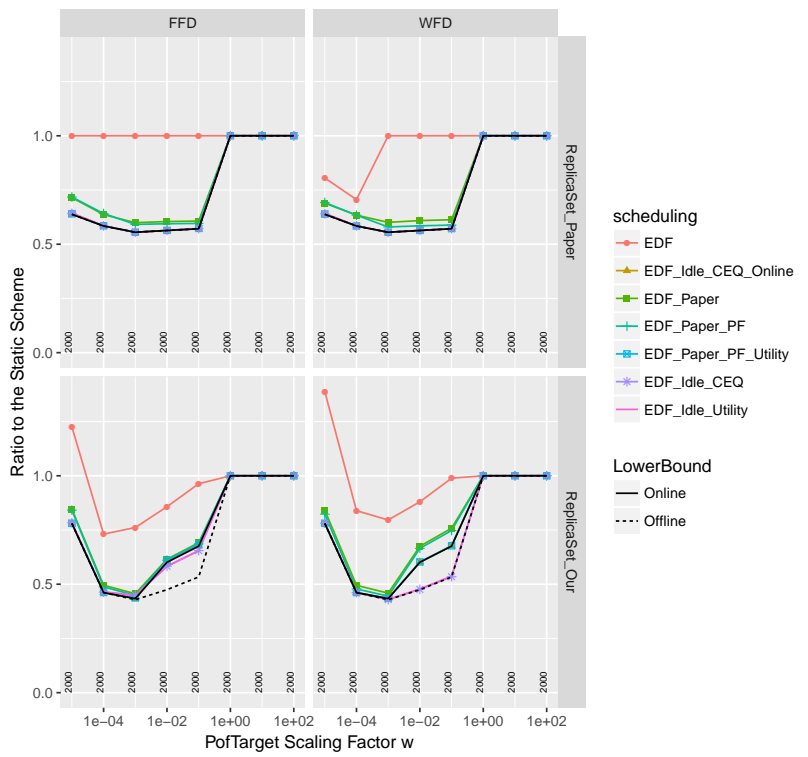

(a) $U_{t o t}=0.5$

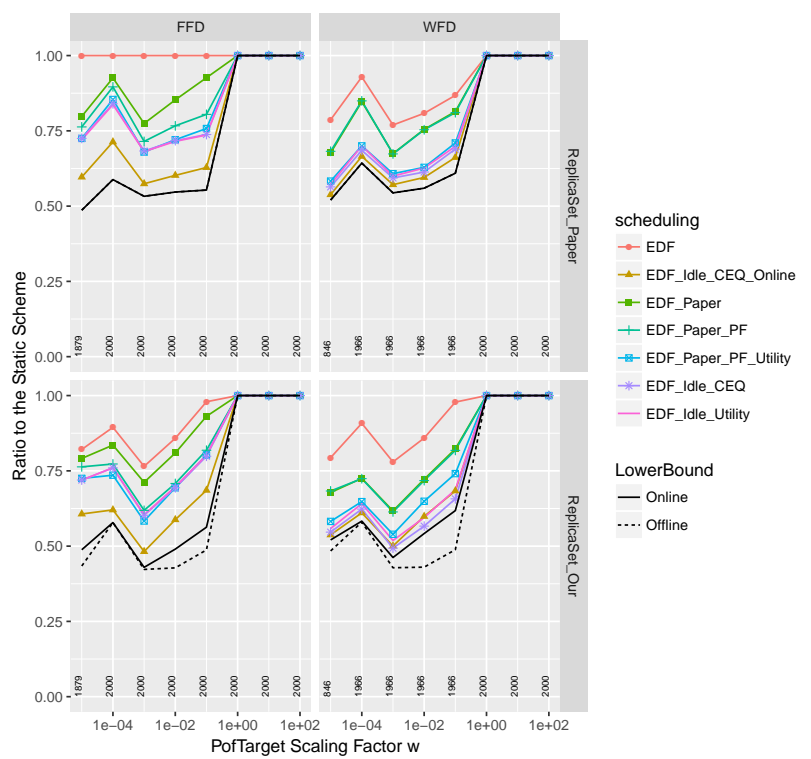

(c) $U_{\text {tot }}=2.5$

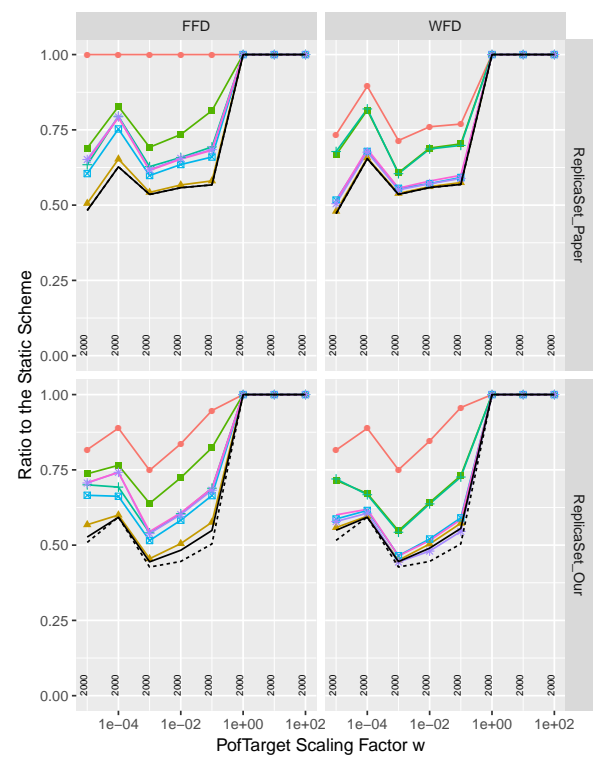

(b) $U_{t o t}=1.5$

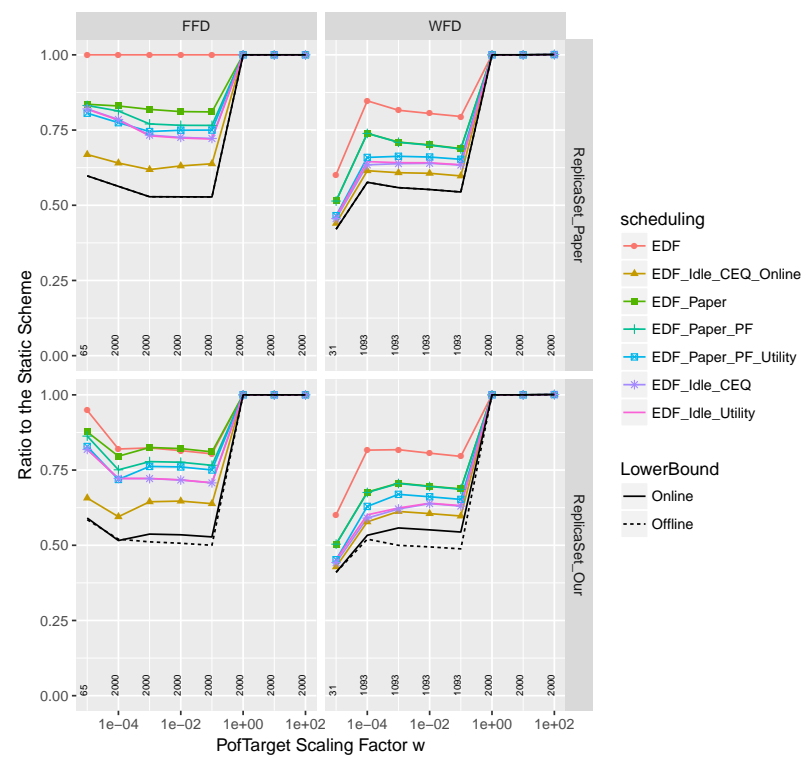

(d) $U_{t o t}=3.5$ scheduling

- EDF

^-EDF_Idle_CEQ_Online

- EDF_Paper

+ EDF_Paper_PF

\#- EDF_Paper_PF_Utility

*-EDF_Idle_CEQ

- EDF_Idle_Utility

LowerBound

- Online

Figure 13: Impact of $U_{t o t}$ and $w$ with $B C / W C=1$ and LEF 


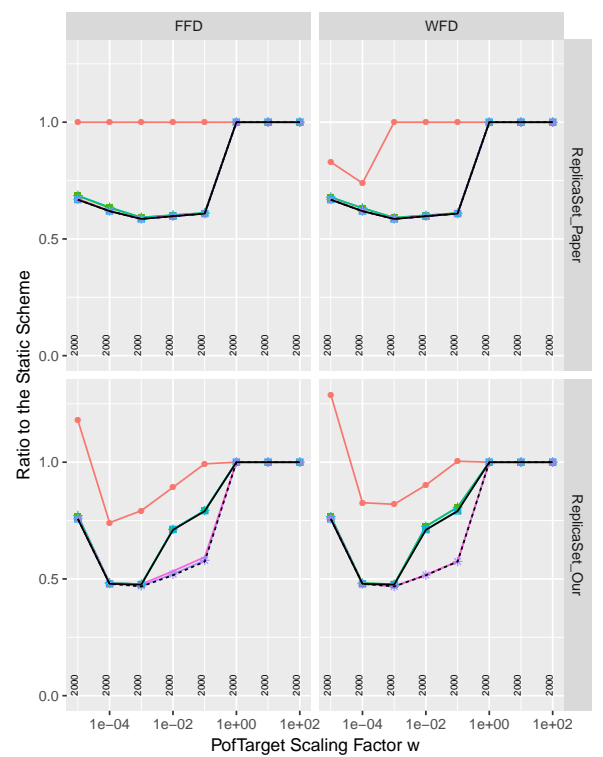

(a) $B C / W C=0.2$

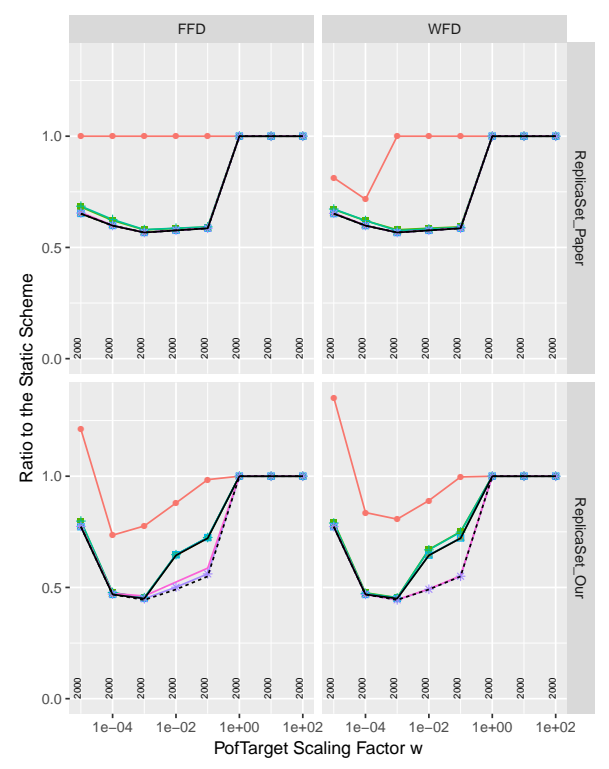

(c) $B C / W C=0.6$

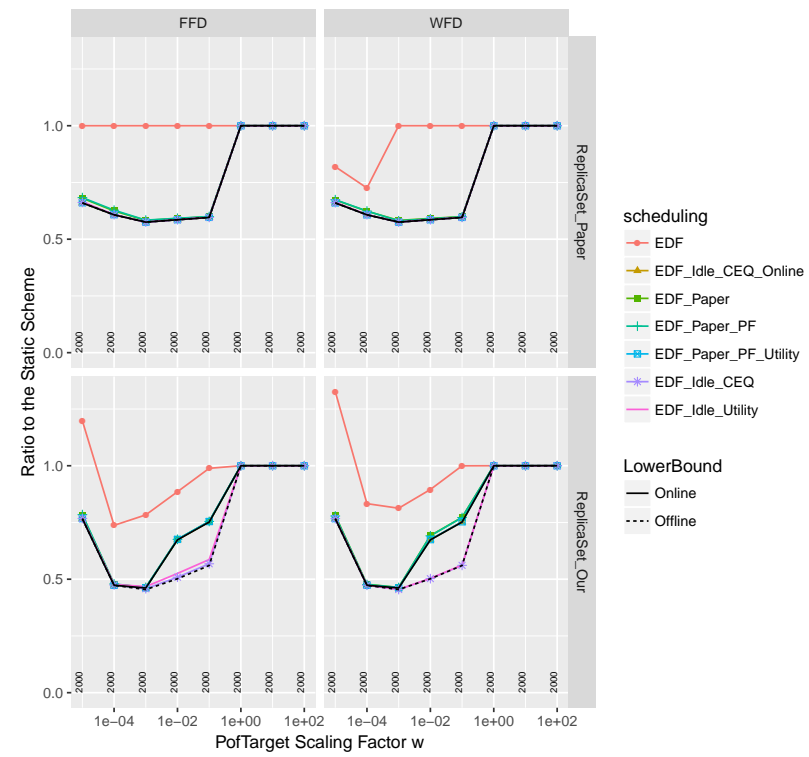

(b) $B C / W C=0.4$

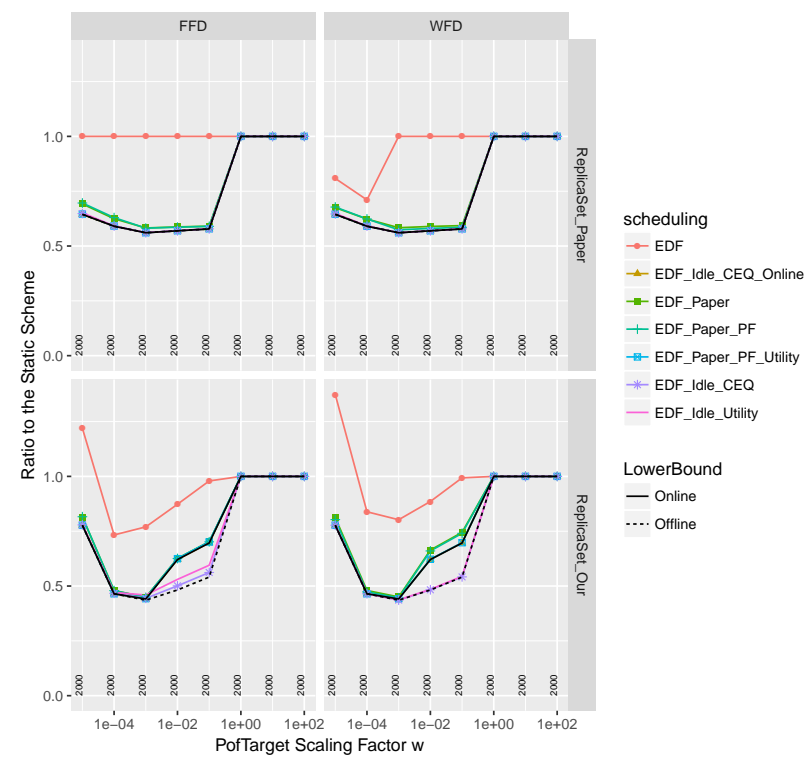

(d) $B C / W C=0.8$

Figure 14: Impact of $B C / W C$ and $w$ with $U_{t o t}=0.5$ and LEF 


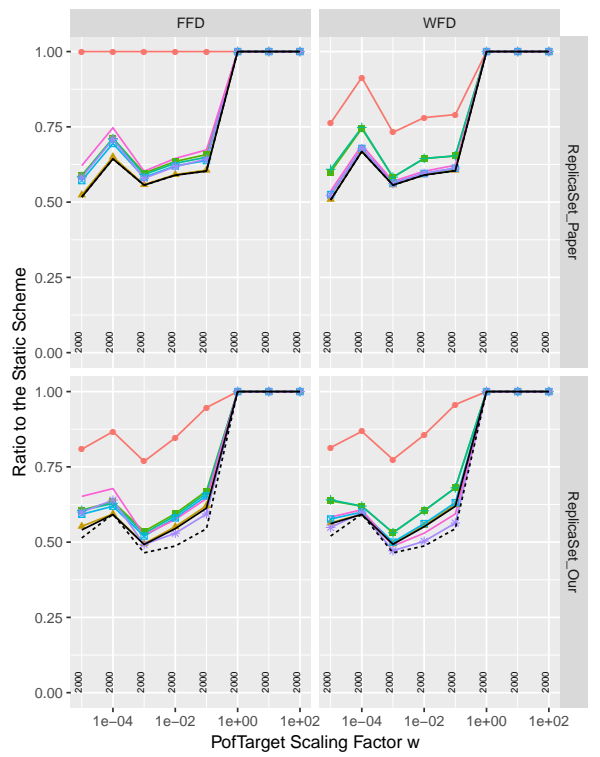

(a) $B C / W C=0.2$

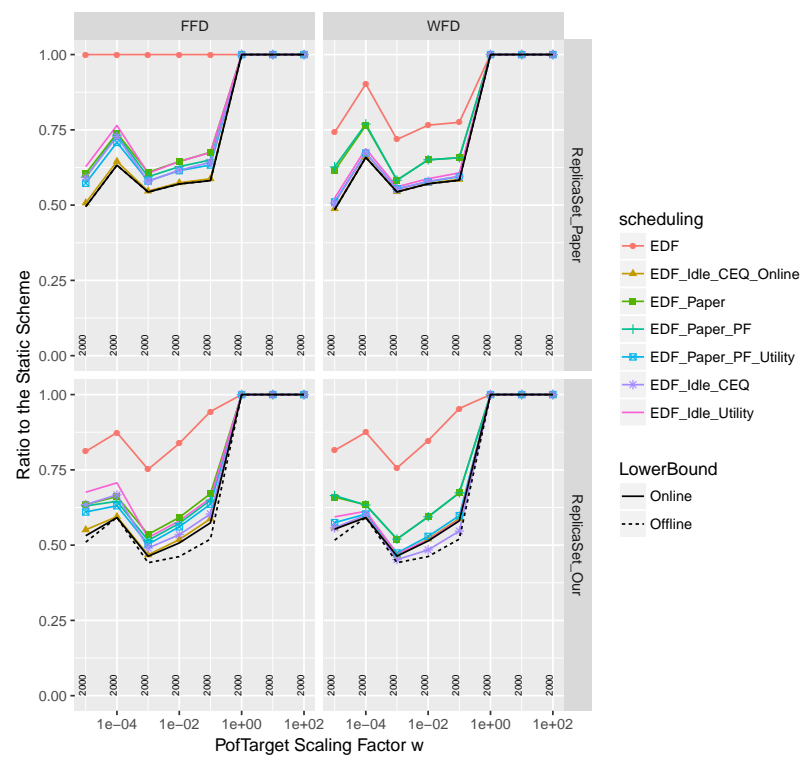

(c) $B C / W C=0.6$

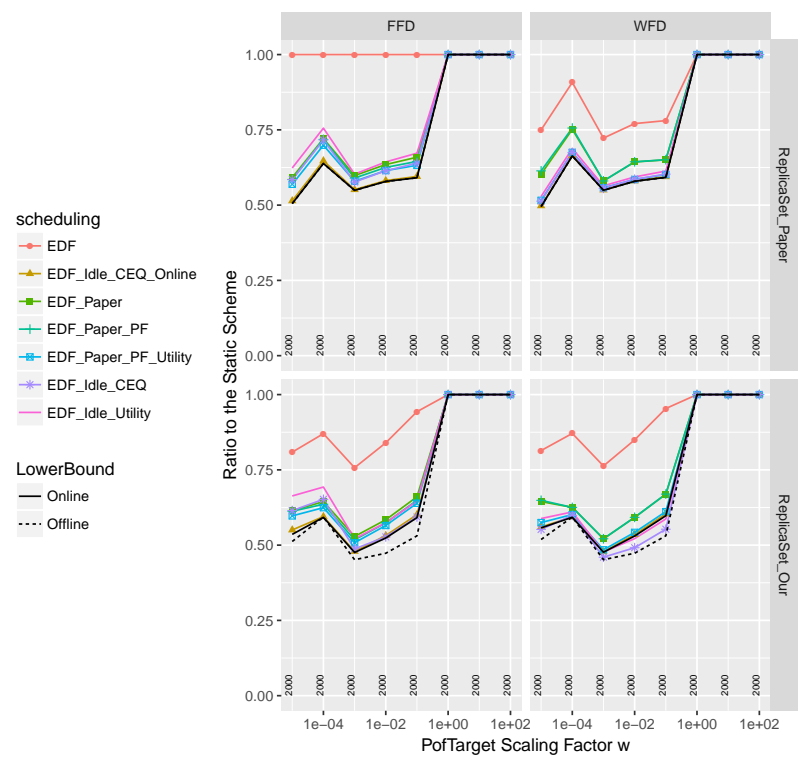

(b) $B C / W C=0.4$

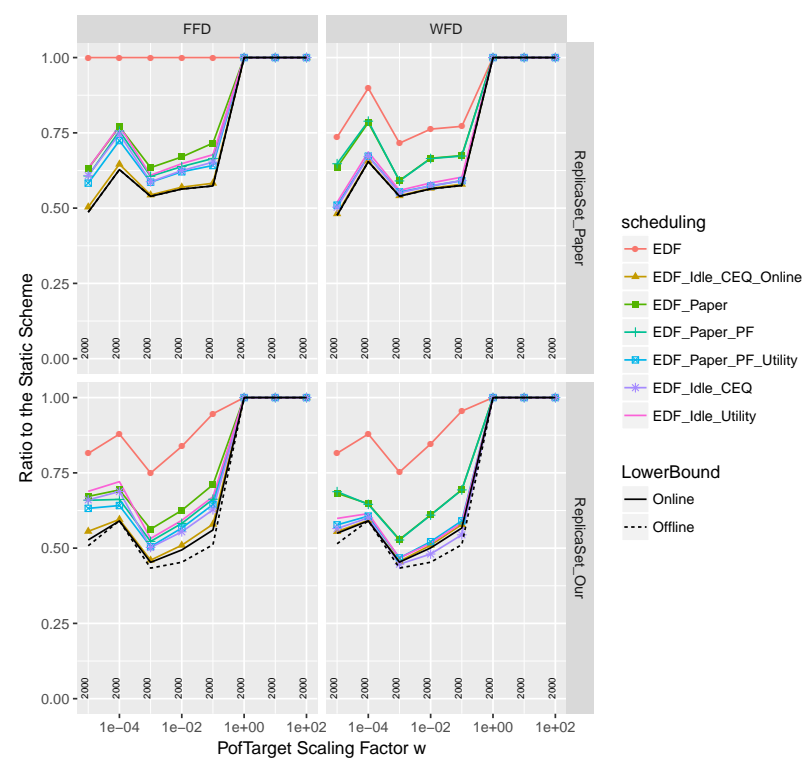

(d) $B C / W C=0.8$ scheduling

$\rightarrow$ EDF

4-EDF_Idle_CEQ_Online

- EDF_Paper

+ EDF_Paper_PF

\#-EDF_Paper_PF_Utility

*-EDF_ddle_CEQ

-EDF_Idle_Utility

LowerBound

— Online

-... Offline

Figure 15: Impact of $B C / W C$ and $w$ with $U_{t o t}=1.5$ and LEF 


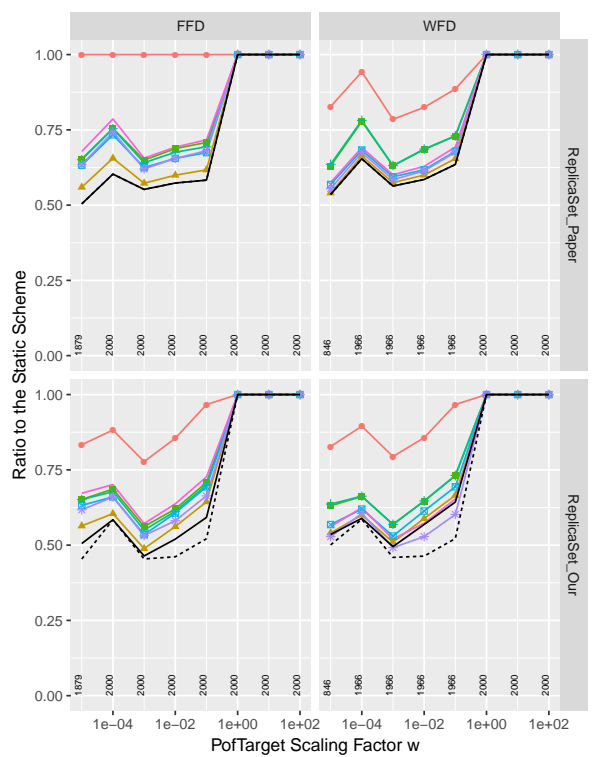

(a) $B C / W C=0.2$

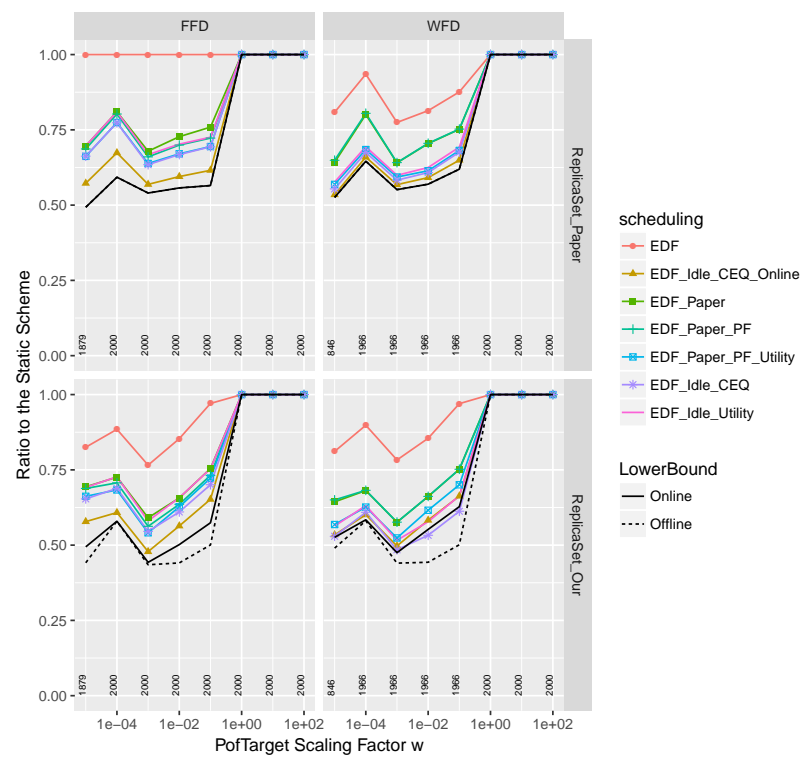

(c) $B C / W C=0.6$

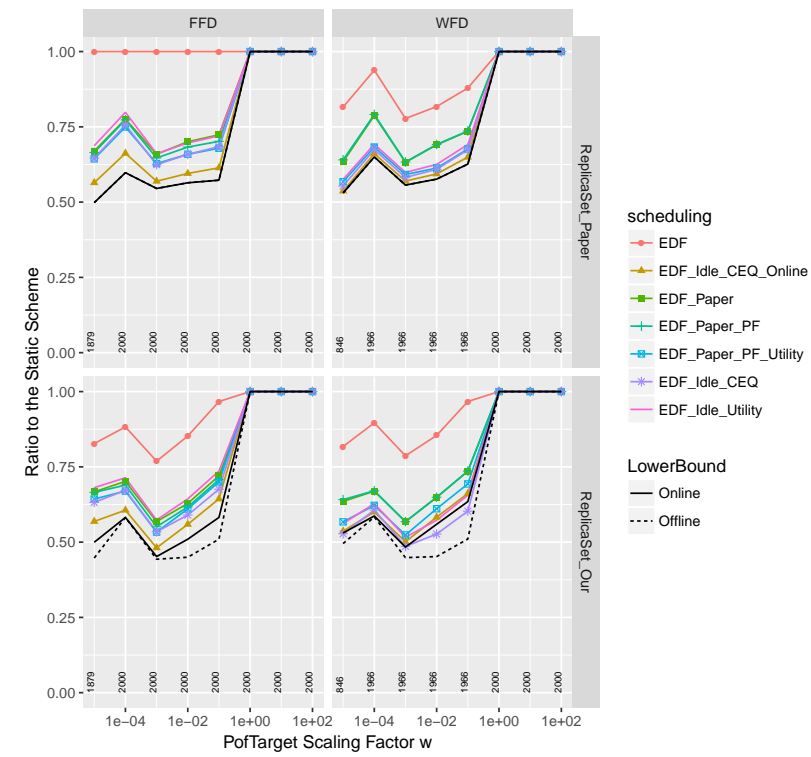

(b) $B C / W C=0.4$

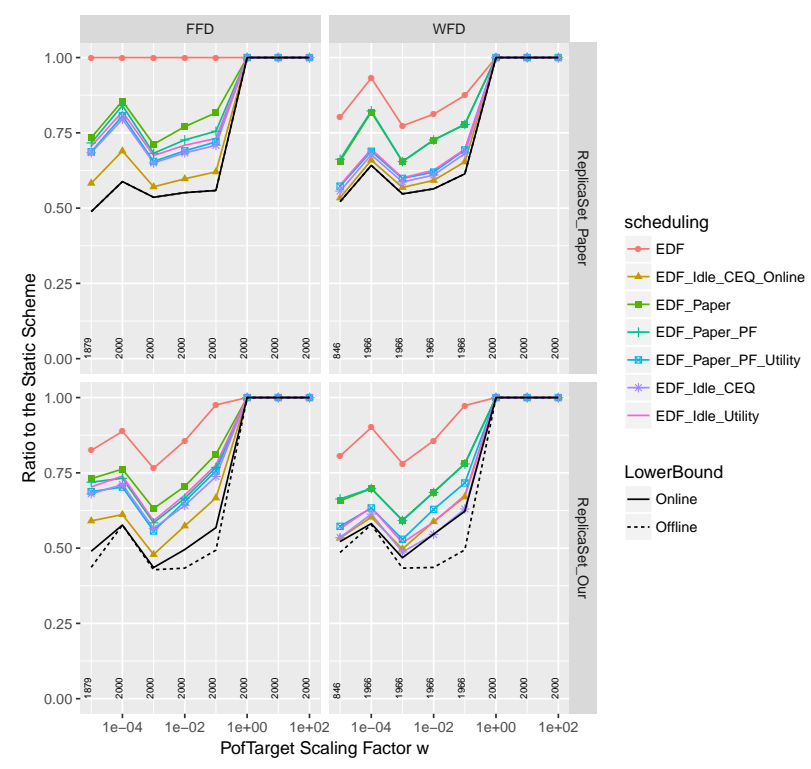

(d) $B C / W C=0.8$

Figure 16: Impact of $B C / W C$ and $w$ with $U_{t o t}=2.5$ and LEF 


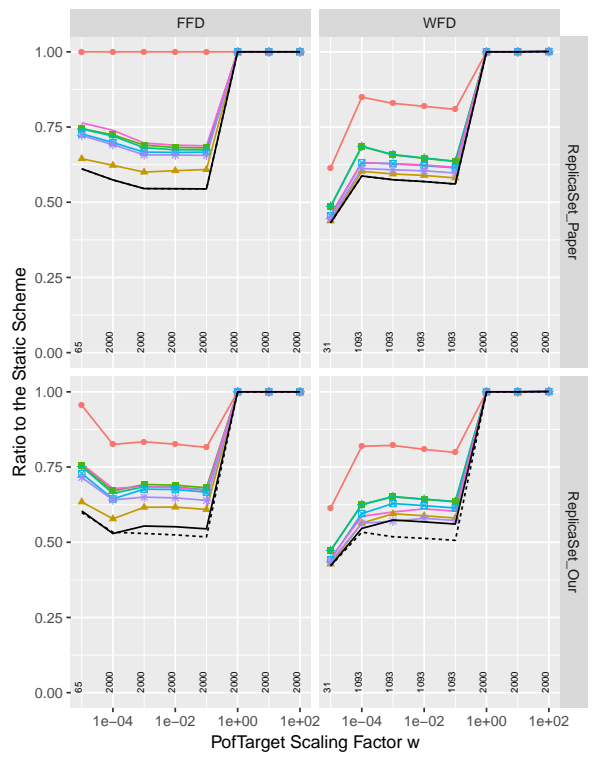

(a) $B C / W C=0.2$

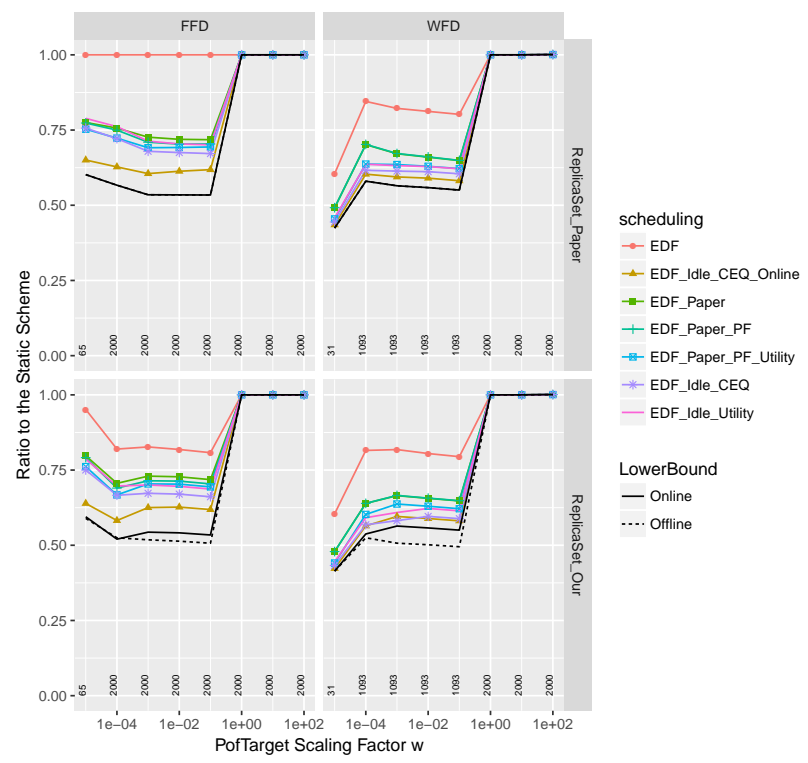

(c) $B C / W C=0.6$

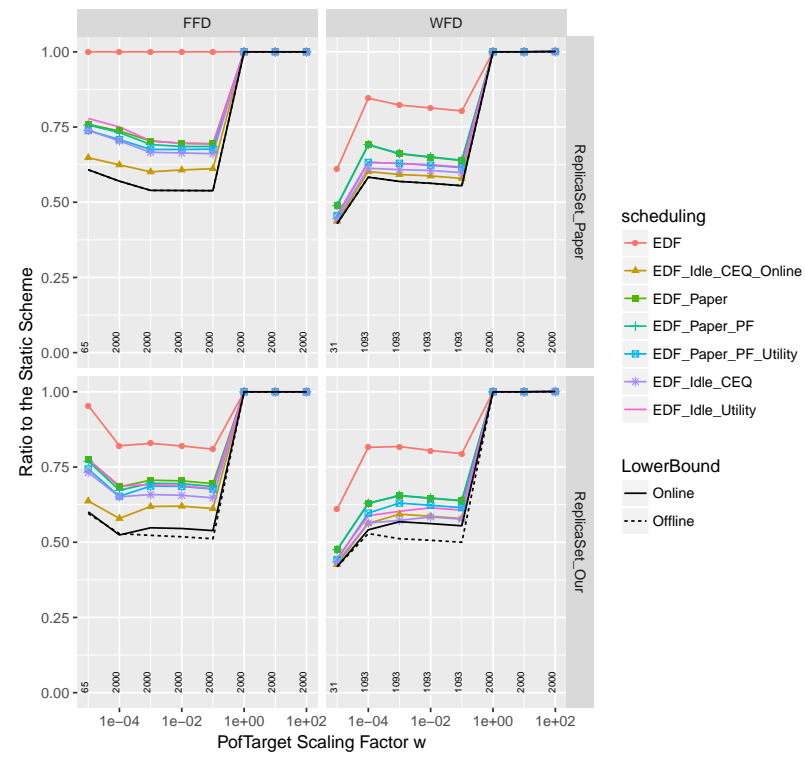

(b) $B C / W C=0.4$

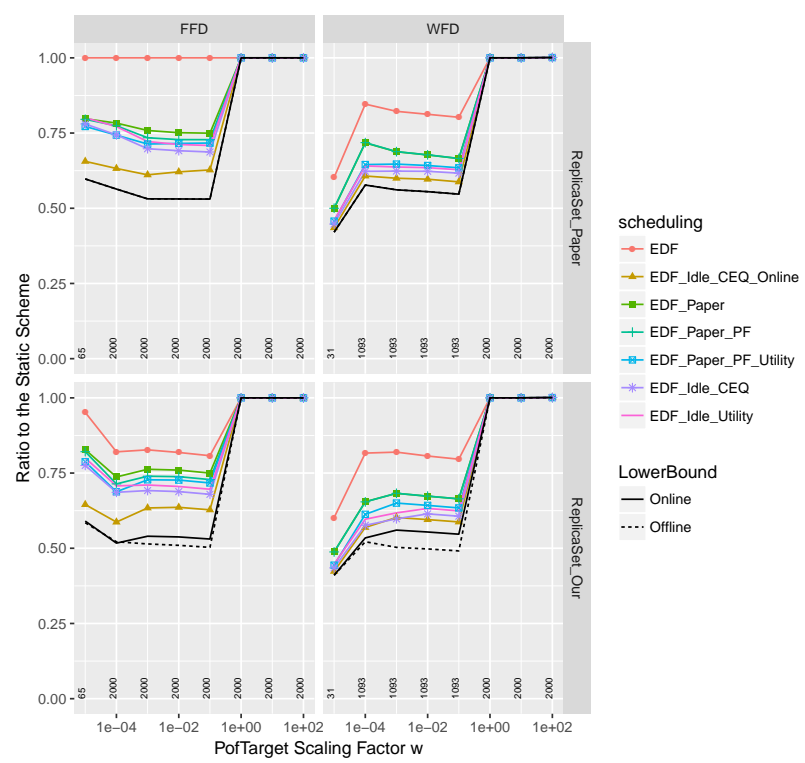

(d) $B C / W C=0.8$

Figure 17: Impact of $B C / W C$ and $w$ with $U_{t o t}=3.5$ and LEF 
bility goes down when the $U_{\text {tot }}$ increases, EDF_IDLE_CEQ_ONLINE catches up and becomes the best. EDF_IDLE_CEQ_ONLINE could save up to $25 \%$ more with respect to EDF_PAPER (see Fig. 8c). It should be noted that when $w \geq 1$ (rightmost parts of graphs), all scheduling heuristics have the same performance as only one replica is needed for each task to meet the reliability target: they all pay the same minimum energy cost. As we decrease the value of $w$, more than one replica is needed, so we can clearly see the difference on capability to reduce the overlapping with several scheduling heuristics. In Figure 8, we keep the value of $B C / W C$ at 1 and increase the system load. We observe that, the energy savings are closer to the lower bound at lower utilization, as we can find feasible partitioning without overlapping for the minimum energy configurations. In Figure 10, for a fixed system load $\left(U_{t o t}=1.5\right)$, the higher $B C / W C$ ratio, the lower workload variability, and the further to the lower bound. This is because as $B C / W C$ increases, jobs have larger execution time and there is a higher chance of overlap between the primary and the secondary replicas.

Another common trend is that, in the majority of cases, WFD and RePlicaSet_Our help saving energy. By applying ReplicaSet_Our, we could find a lower $f_{\text {opt }(i)}$ in most of the cases. Remind that REPLICASET_OUR adds up one replica at $f_{i}$ and $x$ replicas at $f_{\text {max }}$ while REPLICASET_PAPER adds up them all at $f_{i}$, at the end, they may find a different $f_{\text {opt }(i)}$. By considering WFD, we could be able to: 1) achieve the highest energy saving, 2) improve the average performance of scheduling heuristics. For example, in subfigure 10a, from the two plots in the first row (with the same replica set but different mapping), we can see that more scheduling heuristics could achieve the lower bound with WFD compared to FFD (only EDF_IDLE_CEQ_OnLine did). From the second row, we could see that the best performance is EDF_IDLE_CEQ with WFD. Applying the the competitor's replica sets and mapping (top left plot), the best energy saving is achieved by EDF_IDLE_CEQ_ONLINE around 45\%. With our replica sets and mapping (bottom right), all our scheduling heuristics could achieve more than 50\% energy saving. Even the classic EDF could save up to 25\%. These improvements are representative of the trends that can be observed for all considered graphs, but suffer from some exceptions. For example, in Fig. 8a, when $w=10^{-5}$, REPLiCASET_OUR gives a higher lower bound which means we will lose even without any overlapping in the schedule. Overall, our task mapping and replica set never achieves significantly bad performance, and most of the time achieves the best performance. Altogether, the average improvement of our method over [9], computed across all our experiments, can be estimated of the order of $20 \%$. 


\section{Related work}

Liu and Layland first introduced the Earliest Deadline First (EDF) and the Rate Monotonic (RM) scheduling policies for real-time systems and provided the utilization bounds for both policies in 1973 [11]. Since then, the real-time scheduling problem has been extensively studied. A significant amount of work has aimed at developing new scheduling algorithms under different assumptions, including the well-known energy management technology Dynamic Voltage and Frequency Scaling (DVFS). We refer to the original article [9] for the related work. Here, we only cover relevant work citing paper [9].

In [13], Taherin et al. propose an approach for energy management that is only applied on "low-criticality tasks in low-criticality mode to preserve the original reliability of the system". This approach cannot guarantee that a reliability threshold is met. Keeping the maximum power consumption below the chip thermal design power, Ansari et al. [1] have proposed a peak power management approach to meet thermal design power in faulttolerant system. However, in the scheduling task graph, all tasks have the same period/deadline. Cao et al. in [5] proposed an affinity-driven modeling and scheduling approach to makespan optimization. They optimize average peak temperature and makespan, but not energy minimization. To improve quality in real-time system, Cao et al. in [4] proposed QoS-adaptive approximate real-time computation optimization. Approximate results of tasks are allowed: each task is composed of a mandatory part and an optional one that refines the result of the mandatory task. However, failures are not considered. Zhou et al. consider in [15] both transient and permanent faults. They try to improve soft-error reliability while satisfying a lifetime reliability constraint, but do not attempt to minimize energy consumption.

\section{Conclusion}

In this work, we have revisited the challenging problem presented by Haque, Aydin and Zhu in [9], namely minimizing the expected energy consumption of a set of preemptive periodic real-time tasks, executing on a parallel platform whose processors are subject to transient failures. Replication is used to enforce all deadlines, as well as the reliability threshold. We have improved the approach of [9] as follows. First, we use a different formula to estimate the energy consumption, which is supposed to be closer to actual execution scenarios. Secondly, in the mapping phase, we apply a layered WFD strategy, which is expected to be helpful for load-balancing, and for decreasing the overlap among copies of the same task. Finally, we implement several novel scheduling strategies, which introduce the idea of reordering chunks in between deadlines and of taking advantage of the utilization of the 
processor. Moreover, we have established that the sole problem of scheduling tasks with different WCETs, knowing the number of replicas, frequency and assigned processor for each task, is NP-complete in the strong sense.

We have evaluated the improvement of our strategies with a discrete event simulator (made publicly available). Extensive experiments conducted for various range of parameters have shown that: 1) REPLICASET_OuR and WFD help finding a lower frequency, which gives good pre-condition for further energy savings; 2) our scheduling heuristics significantly outperform EDF_PAPER. More specifically, EDF_IDLE_CEQ and EDF_IDLE_CEQ_ONLINE have the best performance under a wide range of scenarios, with an average gain of $20 \%$.

Short-term future work will be devoted to extending the heuristics presented in this paper. It could be interesting to adapt the reordering mechanism from idleness-based methods to CEQ-based methods as it is done with both EDF_IDLE_CEQ and EDF_IDLE_CEQ_ONLINE, but with scaled WCET in the CEQ. Moreover, Proposing an online version for each newly introduced method remains to be achieved. On the long term, future work will aim at extending the algorithms to periodic workflows instead of independent task sets. The dependencies between nodes will dramatically complicate the problem. Another interesting direction is to deal with the same problem with independent tasks, but targeting heterogeneous multicore systems.

\section{References}

[1] M. Ansari, S. Safari, A. Y. Khaksar, M. Salehi, and A. Ejlali. Peak power management to meet thermal design power in fault-tolerant embedded systems. IEEE Transactions on Parallel and Distributed Systems, 2018.

[2] H. Aydin and Q. Yang. Energy-aware partitioning for multiprocessor real-time systems. In Proc. 17th Int. Symp. Parallel and Distributed Processing, IPDPS '03. IEEE Computer Society, 2003.

[3] E. Bini and G. C. Buttazzo. Measuring the performance of schedulability tests. Real-Time Systems, 30(1-2):129-154, 2005.

[4] K. Cao, G. Xu, J. Zhou, T. Wei, M. Chen, and S. Hu. Qos-adaptive approximate real-time computation for mobility-aware iot lifetime optimization. IEEE Transactions on Computer-Aided Design of Integrated Circuits and Systems, pages 1-1, 2018.

[5] K. Cao, J. Zhou, P. Cong, L. Li, T. Wei, M. Chen, S. Hu, and X. S. Hu. Affinity-driven modeling and scheduling for makespan optimiza- 
tion in heterogeneous multiprocessor systems. IEEE Transactions on Computer-Aided Design of Integrated Circuits and Systems, 2018.

[6] E. G. Coffman, Jr., M. R. Garey, and D. S. Johnson. Approximation algorithms for bin packing: A survey. In D. S. Hochbaum, editor, Approximation Algorithms for NP-hard Problems, pages 46-93. PWS Publishing Co., 1997.

[7] M. R. Garey and D. S. Johnson. Computers and Intractability, a Guide to the Theory of NP-Completeness. W.H. Freeman and Company, 1979.

[8] L. HAN. Resilience for energy-aware periodic real-time systems - Simulator, 22019.

[9] M. A. Haque, H. Aydin, and D. Zhu. On reliability management of energy-aware real-time systems through task replication. IEEE Transactions on Parallel and Distributed Systems, 28(3):813-825, 2017.

[10] D. Johnson, A. Demers, J. Ullman, M. Garey, and R. Graham. Worstcase performance bounds for simple one-dimensional packing algorithms. SIAM Journal on Computing, 3(4):299-325, 1974.

[11] C. L. Liu and J. W. Layland. Scheduling algorithms for multiprogramming in a hard-real-time environment. Journal of the ACM (JACM), 20(1):46-61, 1973.

[12] A. Schrijver. Combinatorial Optimization: Polyhedra and Efficiency, volume 24 of Algorithms and Combinatorics. Springer-Verlag, 2003.

[13] A. Taherin, M. Salehi, and A. Ejlali. Reliability-aware energy management in mixed-criticality systems. IEEE Transactions on Sustainable Computing, 2018.

[14] B. Zhao, H. Aydin, and D. Zhu. Energy management under general task-level reliability constraints. In 18th Real-Time and Embedded Technology and Applications Symposium (RTAS), pages 285-294. IEEE, 2012.

[15] J. Zhou, T. Wei, M. Chen, X. S. Hu, Y. Ma, G. Zhang, and J. Yan. Variation-aware task allocation and scheduling for improving reliability of real-time mpsocs. In DATE, pages 171-176, 2018. 


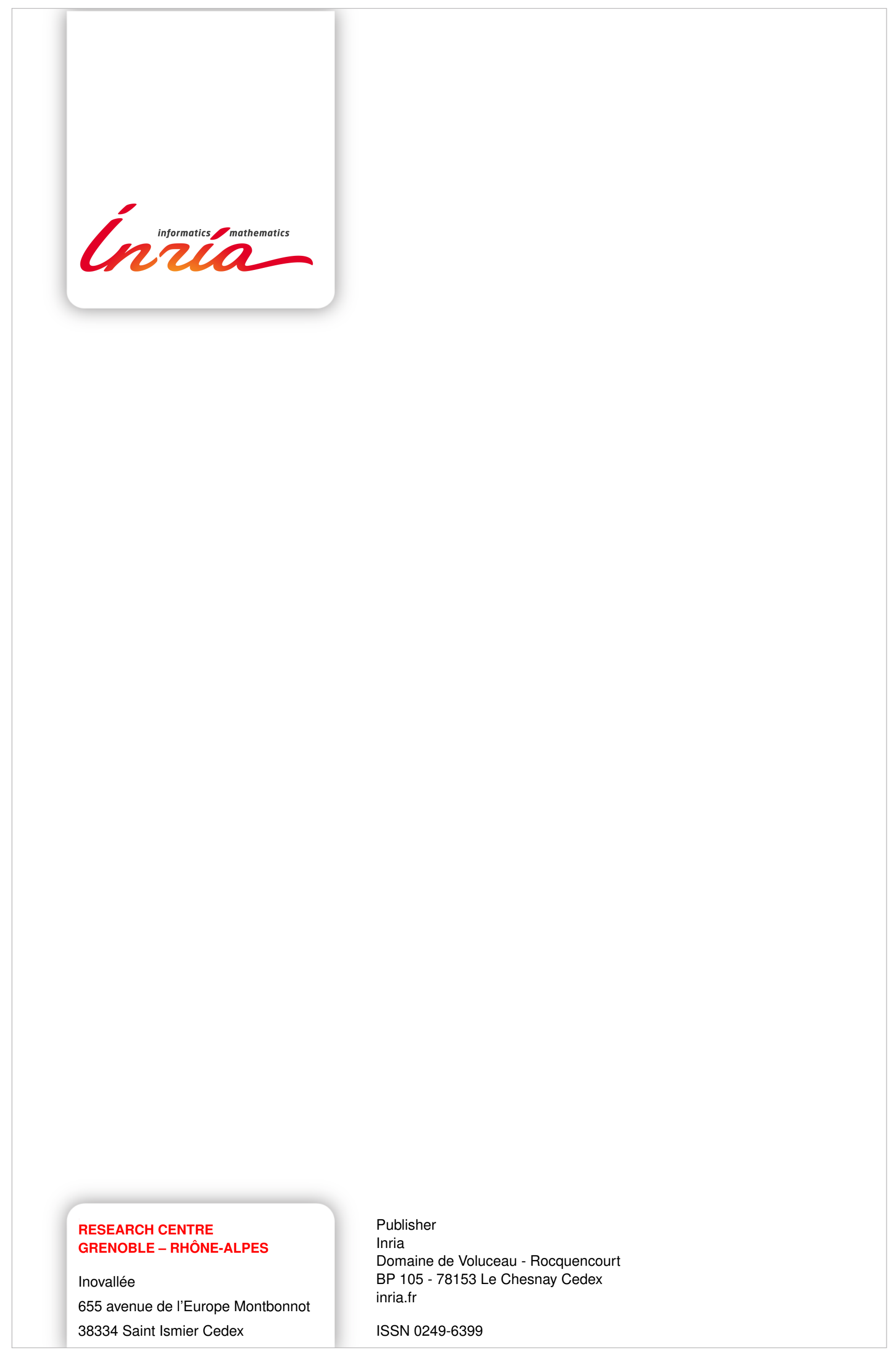

